\title{
Solar wind originating in funnels: fast or slow?
}

\author{
Å. M. Janse ${ }^{1,4}, \varnothing$. Lie-Svendsen ${ }^{2,1}$, and E. Leer ${ }^{1,3,4}$ \\ ${ }^{1}$ Institute of Theoretical Astrophysics, University of Oslo, PO Box 1029, Blindern, 0315 Oslo, Norway \\ e-mail: a.m.janse@astro.uio.no \\ 2 Norwegian Defence Research Establishment, PO Box 25, 2027 Kjeller, Norway \\ 3 High Altitude Observatory, National Center for Atmospheric Research, Boulder, Colorado, USA \\ 4 Centre of Mathematics for Applications, University of Oslo, Norway \\ Received 29 August 2006 / Accepted 26 July 2007
}

\section{ABSTRACT}

\begin{abstract}
Aims. We model a hydrogen-helium solar wind originating in funnels, regions of rapid flux tube expansion at the base of the solar corona.

Methods. The time-dependent model describes the particle density, flow speed, temperature parallel and perpendicular to the magnetic field, and the heat flow for each ionization state of hydrogen and helium, and for electrons.

Results. For a large range of heating parameters, the funnel has two co-existing solutions: both a slow and a fast solar wind solution result from the same heating parameters, depending on the initial state from which the model was started. Though the fast and the slow solar wind can co-exist it is difficult to change from a fast solar wind to a slow solar wind or vice versa. A significant change in the heating parameters is required to "flip" the solution, and it takes a long time, about one month, to reach the other steady state solution. When either the funnel or helium is removed from the model, we no longer have two co-existing states.
\end{abstract}

Key words. solar wind - Sun: corona - Sun: abundances - Sun: transition region - hydrodynamics

\section{Introduction}

Over the last decade there have been several studies indicating that the fast solar wind may have its origin in coronal holes with "funnels," a region with rapid flux tube expansion at the base of the corona. These magnetic field structures were suggested by Gabriel (1976) and Dowdy et al. (1986) and later discussed by Rabin (1991) and others. Axford \& McKenzie (1992) suggested that the solar wind may originate in regions of strong magnetic field, and these ideas were also discussed by Axford $\&$ McKenzie (1997). Since then there have been quite a few studies of the plasma flow in solar funnels: Hackenberg et al. (2000) studied the solar wind flow in funnels, at the base of polar coronal holes, and Li (2002) studied heating of an electronproton- $\alpha$-particle solar wind in coronal funnels by ion cyclotron waves. Esser et al. (2005) studied a hydrogen solar wind in coronal holes, with and without funnels, and compared the Ly $\alpha$ radiation in these models. The model with funnels produced Ly $\alpha$ radiation in better agreement with observations than did the "pure coronal hole" model. For an overview of the solar wind originating in funnels, see Tu et al. (2005) and references therein.

Since the funnel is located below the region where most of the energy driving the solar wind must be deposited, one may ask whether the funnel indeed matters for the solar wind. If the coronal plasma is largely unaffected by the magnetic field structure below the corona, one could expect that the solar wind also will not depend sensitively on this structure. On the other hand one may ask whether a narrow funnel will be able to supply the observed mass flux, which will require a rapid flow in the upper chromosphere and transition region. And one may question the assumption that the corona is unaffected by the presence of a funnel at the base since the coronal density is closely linked to the energy balance of the transition region - corona - solar wind system.
The energy flux conducted downwards from the corona to the transition region has to be large enough to supply the particle flux with the needed energy to heat the upstreaming plasma from chromospheric to coronal temperatures. Neglecting helium and assuming that protons and electrons have the same coronal temperature $T_{\mathrm{c}}$, the energy flux density needed to be conducted into the transition region in order to sustain a solar wind proton particle flux density at Earth of $\left(n_{\mathrm{p}} u_{\mathrm{p}}\right)_{\mathrm{E}}$ (where $n_{\mathrm{p}}$ and $u_{\mathrm{p}}$ are the proton density and flow speed) is $5 k T_{\mathrm{c}}\left(n_{\mathrm{p}} u_{\mathrm{p}}\right)_{\mathrm{E}} A_{\mathrm{E}} / A_{\mathrm{c}}$, where $k$ is Boltzmann's constant and $A_{\mathrm{c}}$ and $A_{\mathrm{E}}$ are the flow tube area in the corona (above the funnel) and at Earth, respectively. Conducting a given energy flux into a funnel requires a higher coronal temperature (because the downward heat flux in a fully ionized plasma is proportional to $T^{5 / 2}$ ) than conducting it into a tube of constant cross section. However, the coronal temperature cannot be increased indefinitely, because the energy loss into the solar wind increases as the coronal temperature is increased, and it is therefore possible that the downward conductive energy flux is not large enough to sustain the solar wind particle flux we observe at $1 \mathrm{AU}$.

The funnel geometry can also produce the opposite effect, however: in a radiation-dominated transition region the pressure is directly proportional to the electron heat flux density, $q_{\mathrm{e}}$, from the corona. A given downward heat flux $A q_{\mathrm{e}}$ in a funnel with a rapidly decreasing cross section $A$ could produce a rapidly increasing heat flux density $q_{\mathrm{e}} \propto 1 / A$ and consequently a high transition region pressure. As the coronal pressure is close to the transition region pressure, this would lead to an increase in the coronal density and enhanced collisional coupling in the corona. The end result could be a high radiation loss, coronal densities much higher than what is observed in coronal holes, and difficulties producing a high-speed wind with a reasonable mass flux.

A long-standing problem of solar wind modelling is that coronal hole electron densities inferred from observations are 
typically a factor 10 higher than in models of the fast solar wind, and, conversely, models that reproduce observed coronal hole densities tend to produce a solar wind with a higher mass flux and a lower terminal wind speed than what is observed in the fast solar wind (see Lie-Svendsen et al. 2003). Since the energy balance of a wind originating in funnels may conceivably be quite different from the energy balance without the funnel at the base, it is worthwhile to investigate whether a funnel may resolve, or alleviate, this problem.

Of the minor elements helium is important because its abundance is so high in the solar atmosphere that it may affect the corona-solar wind system. Helium will easily be forced up through the transition region and into the corona, while removing helium from the corona requires an $\alpha$-particle temperature that is much higher than the proton temperature. If this is not the case, as will happen in a high-density corona with strong collisional coupling to protons, the coronal helium density can easily become comparable to the proton density, in which case the solar wind acceleration is strongly affected by helium (Leer et al. 1992; Hansteen et al. 1997). In solar wind models without a funnel at the base, another problem has been to understand how a sufficiently high helium abundance is maintained in the upper chromosphere. The frictional drag from hydrogen is insufficient to maintain a fairly well-mixed chromosphere, and without some other (unknown) process to maintain the helium abundance, gravitational settling in the chromosphere causes the solar wind helium abundance to be many orders of magnitude smaller than what is observed (Lie-Svendsen et al. 2003).

One advantage of including a funnel at the base of the coronal hole is that helium will not settle in the chromosphere. The frictional force acting on neutral helium from neutral hydrogen is large enough to increase the helium scale length to that of hydrogen. The neutral hydrogen pressure balances the gravitational force. For the four times heavier helium, the pressure can only balance $1 / 4$ of the gravitational force, when hydrogen and helium have the same scale height. Hence the frictional force on neutral helium has to be $3 / 4$ of gravity to maintain a constant helium abundance in the upper part of the chromosphere. The frictional force is proportional to the drift speed difference of the colliding particles. As the funnel has a small cross section, the chromospheric flow speed has to be high, for a given solar wind particle flux, and the relative difference in flow speed between hydrogen and helium is small.

When the helium flow speed in the chromosphere is close to the hydrogen flow speed, as it then must be in funnels with a sufficiently small cross section, the ratio between the solar wind flux of $\alpha$-particles and protons is almost equal to the helium abundance in the chromosphere. Since the relative flow speed difference between protons and $\alpha$-particles at $1 \mathrm{AU}$ is small, this also implies that the solar wind helium abundance must equal the chromospheric helium abundance. In other words, the strong coupling between hydrogen and helium in a fast flowing funnel implies that the flux of helium is locked to the hydrogen flux.

Not only will the funnel flow ensure that helium does not settle in the chromosphere, but it also implies that helium must flow into the corona (and, in a steady state, into the solar wind) at a rate equal to the chromospheric abundance times the hydrogen flux. If helium, streaming into the corona, is not given sufficient energy to escape the solar gravitational field, it builds up in the corona. Eventually the helium flux into the corona must be reduced in order to restore the balance between inflow and outflow from the corona. Since the flux is locked to the hydrogen flux, this does not happen until the helium abundance becomes so high that helium is able to reduce the flow of hydrogen. We may therefore end up with more helium than hydrogen in the corona and a solar wind that is very different from an electronproton solar wind with a few percent helium everywhere.

In order to address these issues, a model must obviously span the region from the chromosphere and into the supersonic solar wind, and radiative loss in the transition region must be accounted for since this affects the density of the corona. In this type of radiative energy balance model the structure of the corona and the solar wind outflow are largely determined by the flow geometry and the coronal heat input (Withbroe 1988). It is therefore important to describe the heat flow from the corona into the transition region as correctly as possible. In addition, the sharp temperature increase in the transition region leads to a "thermal" force acting on minor ions (Nakada 1969). This force is a collisional effect: the Coulomb cross section is inversely proportional to the relative velocity of the colliding particles to the fourth power, so a heavy ion (for instance an $\alpha$-particle) will collide less frequently with the downflowing hot protons than the cold upward moving protons. This results in a net upward force which may become larger than gravity, and therefore needs to be properly accounted for in the model.

The present study is based upon a newly developed set of gyrotropic transport equations (Janse et al. 2005). These are based on a bi-Maxwellian velocity distribution function with a correction term which is of third order in velocity. This set reduces to the equations for an isotropic plasma $\left(T_{\perp}=T_{\|}\right)$developed by Killie et al. (2004). The transport coefficients, in the collisiondominated limit, agree quite well with classical transport theory (see Killie et al. 2004). These equations, which are tailored to a fully ionized gas, were developed because fluid transport equations in common use today (Schunk 1977; Demars \& Schunk 1979) lead to heat flux coefficients and thermal forces that deviate strongly from the results of classical transport theory (e.g. Spitzer \& Härm 1953; Braginskii 1965). The new equations are not more complex than, e.g., the equations of Demars \& Schunk (1979) in the gyrotropic limit, and are therefore well suited to be implemented in a numerical model. The description of the outer, collisionless solar wind is similar to the Demars \& Schunk description and in good agreement with the results from collisionless kinetic models of the evolution of the velocity distribution function.

Janse et al. (2006) studied the outflow of a hydrogen-protonelectron plasma in coronal holes without a funnel at the base, using the transport equations developed by Janse et al. (2005). A major finding of that study was that the heat flux from the corona was so large that there was no need for direct heating of the transition region. This is in contrast to previous studies by Lie-Svendsen et al. (2001, 2002), using the equations of Demars $\&$ Schunk (1979), who found that the inward heat flux from the corona was not large enough to supply the energy needed to heat the upwelling plasma to coronal temperatures and balance the radiative loss from the transition region. It was therefore argued that it is necessary to add heat directly to the transition region to produce a reasonable corona and solar wind.

This work is an extension of the study by Esser et al. (2005), where the same equation set was solved and the same numerical model was used. The main difference is that we now include helium. The study by Esser et al. (2005) established that it is indeed possible to have a realistic fast solar wind with a funnel at its base: sufficient heat can be conducted into the transition region to supply the enthalpy flux necessary for the observed solar wind mass flux, while the radiation loss can be kept sufficiently small to ensure a pressure in approximate agreement with coronal hole observations. Moreover, it was found that the funnel solution, but 
not a solar wind without a funnel at its base, could accommodate both the observed Ly $\alpha$ radiation loss and high electron temperatures in the inner corona. In order to achieve the agreement with observations, "fine tuning" of the heating parameters was required: a significant fraction of the energy had to be deposited in electrons in the inner corona to reproduce the observed electron temperature. To prevent a solar wind mass flux much higher than what is observed, the energy deposition in the corona had to be small in order to keep the coronal proton temperature below about $3 \times 10^{6} \mathrm{~K}$, a temperature that is insufficient to drive a fast solar wind. Consequently, about half of the energy flux had to be deposited in the supersonic solar wind, in the form of Alfvén wave heating.

In the study by Esser et al. (2005) fine tuning of heating parameters was necessary since the aim was to determine whether observed properties of the solar wind could be reconciled with the observed Ly $\alpha$ radiation from the Sun. The present study has a different aim, focusing on how helium affects the partitioning of the solar wind energy budget, for which fine tuning of parameters is not appropriate. Instead we shall use a simple exponential damping of the prescribed energy flux, allowing precise control of where energy is deposited and the fraction going into each species.

\section{New gyrotropic transport equations}

For a one-dimensional flow along a radial magnetic field, the gyrotropic transport equations of Janse et al. (2005) reduce to a 5-moment-approximation, describing density $n_{s}$, drift speed $u_{s}$, parallel temperature $T_{s \|}$, perpendicular temperature $T_{s \perp}$ and heat flow $q_{s}$ along the magnetic field for each particle type $s$. The equations take on the form

$$
\begin{aligned}
\frac{\partial n_{s}}{\partial t}= & -\frac{\partial}{\partial r}\left(n_{s} u_{s}\right)-n_{s} u_{s} \frac{1}{A} \frac{\mathrm{d} A}{\mathrm{~d} r}+\frac{\delta n_{s}}{\delta t} \\
\frac{\partial u_{s}}{\partial t}= & -u_{s} \frac{\partial u_{s}}{\partial r}-\frac{k}{m_{s}} \frac{\partial T_{s \|}}{\partial r}-\frac{k T_{s \|}}{n_{s} m_{s}} \frac{\partial n_{s}}{\partial r} \\
& -\frac{1}{A} \frac{\mathrm{d} A}{\mathrm{~d} r} \frac{k}{m_{s}}\left(T_{s \|}-T_{s \perp}\right)+G+\frac{e_{s}}{m_{s}} E+\frac{1}{m_{s} n_{s}} \frac{\delta M_{s}}{\delta t} \\
\frac{\partial T_{s \|}}{\partial t}= & -u_{s} \frac{\partial T_{s \|}}{\partial r}-2 T_{s \|} \frac{\partial u_{s}}{\partial r}-\frac{1}{n_{s} k} \frac{\partial q_{s \|}}{\partial r}-\frac{1}{A} \frac{\mathrm{d} A}{\mathrm{~d} r} \frac{q_{s \|}}{n_{s} k} \\
& +\frac{2}{A} \frac{\mathrm{d} A}{\mathrm{~d} r} \frac{q_{s \perp}}{n_{s} k}+\frac{1}{n_{s} k} \frac{\delta E_{s \|}}{\delta t}+\frac{1}{n_{s} k} Q_{s m \|} \\
\frac{\partial T_{s \perp}}{\partial t}= & -u_{s} \frac{\partial T_{s \perp}}{\partial r}-\frac{1}{A} \frac{\mathrm{d} A}{\mathrm{~d} r} u_{s} T_{s \perp}-\frac{1}{n_{s} k} \frac{\partial q_{s \perp}}{\partial r}-\frac{2}{A} \frac{\mathrm{d} A}{\mathrm{~d} r} \frac{q_{s \perp}}{n_{s} k} \\
& +\frac{1}{n_{s} k} \frac{\delta E_{s \perp}}{\delta t}+\frac{1}{n_{s} k} Q_{s m \perp} \\
\frac{\partial q_{s}}{\partial t}= & -u_{s} \frac{\partial q_{s}}{\partial r}-2 q_{s \|} \frac{\partial u_{s}}{\partial r}-\frac{1}{2} q_{s \|} u_{s} \frac{1}{A} \frac{\mathrm{d} A}{\mathrm{~d} r} \\
& -2 q_{s \perp} \frac{\partial u_{s}}{\partial r}-2 q_{s \perp} u_{s} \frac{1}{A} \frac{\mathrm{d} A}{\mathrm{~d} r} \\
& -\frac{k^{2} n_{s} T_{s \|}}{m_{s}} \frac{\partial}{\partial r}\left(\frac{3}{2} T_{s \|}+T_{s \perp}\right) \\
& -\frac{1}{A} \frac{\mathrm{d} A}{\mathrm{~d} r} \frac{k^{2} n_{s} T_{s \perp}}{m_{s}}\left(T_{s \|}-T_{s \perp}\right)+\frac{\delta q_{s}^{\prime}}{\delta t},
\end{aligned}
$$

where $\delta q_{s}^{\prime} / \delta t$ has been introduced to simplify the equations,

$$
\frac{\delta q_{s}^{\prime}}{\delta t}=\frac{\delta q_{s}}{\delta t}-\frac{k}{m_{s}}\left(\frac{3}{2} T_{s \|}+T_{s \perp}\right) \frac{\delta M_{s}}{\delta t},
$$

and parallel and perpendicular heat flow are given as

$$
\begin{aligned}
q_{s \|} & =\int m_{s} c_{s \|}^{3} f_{s} \mathrm{~d} c_{s} \\
& =30 q_{s} \frac{T_{s \|}^{3}\left(4 T_{s \perp}+3 T_{s \|}\right)}{16 T_{s \perp}^{4}+48 T_{s \perp}^{3} T_{s \|}+6 T_{s \perp}^{2} T_{s \|}^{2}+60 T_{s \perp} T_{s \|}^{3}+45 T_{s \|}^{4}} \\
q_{s \perp} & =\int \frac{m_{s}}{2} c_{s \perp}^{2} c_{s \|} f_{s} \mathrm{~d} c_{s} \\
& =2 q_{s} \frac{T_{s \perp}^{2}\left(8 T_{s \perp}^{2}+24 T_{s \perp} T_{s \|}+3 T_{s \|}^{2}\right)}{16 T_{s \perp}^{4}+48 T_{s \perp}^{3} T_{s \|}+6 T_{s \perp}^{2} T_{s \|}^{2}+60 T_{s \perp} T_{s \|}^{3}+45 T_{s \|}^{4}} .
\end{aligned}
$$

Here $m_{s}$ and $e_{s}$ are the mass and charge of particle $s, t$ is the time, $r$ is the heliocentric distance, $k$ is the Boltzmann' $s$ constant, $G$ is the gravitational acceleration and $E$ is the electric field. $A$ is the area of the flow tube, $A \propto 1 / B$, where $B$ is the magnetic field strength and $Q_{s m \|}, Q_{s m \perp}$ are mechanical heating terms. The collisional terms are given as

$$
\begin{aligned}
& \frac{\delta n_{s}}{\delta t}=\sum_{t}\left(n_{t} R_{t s}-n_{s} R_{s t}\right) \\
& \frac{\delta M_{s}}{\delta t}=-\sum_{t} n_{s} m_{s} v_{s t}\left(u_{s}-u_{t}\right)+\sum_{t} v_{s t} \frac{3}{5} \frac{\mu_{s t}}{k T_{s t}}\left[q_{s}\left(1-\frac{5}{7} \frac{m_{t}}{m_{0}}\right)\right. \\
& \left.-q_{t} \frac{\rho_{s}}{\rho_{t}}\left(1-\frac{5}{7} \frac{m_{s}}{m_{0}}\right)\right]+m_{s} \sum_{t}\left(n_{t} u_{t} R_{t s}-n_{s} u_{s} R_{s t}\right) \\
& -\sum_{t} n_{s} m_{s} v_{s t}^{2 s t r}\left(u_{s}-u_{t}\right) \\
& \frac{\delta E_{s \|}}{\delta t}=-\sum_{t} 2 \frac{m_{s}}{m_{0}} n_{s} v_{s t} k\left\{T_{s \|}-T_{t \|}\right. \\
& \left.-\frac{2}{5}\left[\frac{m_{t}}{m_{s}}\left(T_{s \perp}-T_{s \|}\right)+T_{t \perp}-T_{t \|}\right]\right\} \\
& +\sum_{t}\left(n_{t} k T_{t||} R_{t s}-n_{s} k T_{s \|} R_{s t}\right) \\
& +\frac{2}{3} n_{s} \sum_{t} \mu_{s t} v_{s t}\left(u_{s}-u_{t}\right)^{2} \\
& \frac{\delta E_{s \perp}}{\delta t}=-\sum_{t} 2 \frac{m_{s}}{m_{0}} n_{s} v_{s t} k\left\{T_{s \perp}-T_{t \perp}\right. \\
& \left.+\frac{1}{5}\left[\frac{m_{t}}{m_{s}}\left(T_{s \perp}-T_{s \|}\right)+T_{t \perp}-T_{t \|}\right]\right\} \\
& +\sum_{t}\left(n_{t} k T_{t \perp} R_{t s}-n_{s} k T_{s \perp} R_{s t}\right) \\
& +\frac{2}{3} n_{s} \sum_{t} \mu_{s t} v_{s t}\left(u_{s}-u_{t}\right)^{2} \\
& +n_{s} \sum_{t} \mu_{s t} v_{s t}^{2 s t r}\left(u_{s}-u_{t}\right)^{2} \\
& \frac{\delta q_{s}}{\delta t}=-\sum_{t \neq s} v_{s t}\left\{E_{s t}^{(1)} q_{s}-E_{s t}^{(4)} \frac{\rho_{s}}{\rho_{t}} q_{t}\right. \\
& \left.+\frac{5}{2} p_{s}\left(u_{s}-u_{t}\right)\left[1-\frac{3}{5} \frac{m_{t}}{m_{0}}\right]\right\} \\
& -\frac{16}{35} v_{s s} q_{s}+\sum_{t}\left(q_{t} R_{t s}-q_{s} R_{s t}\right) \text {, }
\end{aligned}
$$


with mass factors

$$
\begin{aligned}
& E_{s t}^{(1)} \equiv \frac{1}{m_{0}^{3}}\left(3 m_{s}^{3}-\frac{1}{2} m_{s}^{2} m_{t}-\frac{2}{5} m_{s} m_{t}^{2}-\frac{4}{35} m_{t}^{3}\right) \\
& E_{s t}^{(4)} \equiv \frac{1}{m_{0}^{3}}\left(\frac{6}{5} m_{t}^{3}-\frac{171}{70} m_{t}^{2} m_{s}-\frac{3}{7} m_{t} m_{s}^{2}\right) .
\end{aligned}
$$

Here $\rho_{s}=m_{s} n_{s}$ is the mass density, $p_{s}=n_{s} k T_{s}$ is the pressure, $m_{0}=m_{s}+m_{t}$ is the total mass and $\mu_{s t}=\left(m_{s} m_{t}\right) /\left(m_{s}+m_{t}\right)$ is the reduced mass. $T_{s t}=\left(T_{s} m_{t}+T_{t} m_{s}\right) /\left(m_{s}+m_{t}\right)$ is the "reduced" temperature, $T_{s}=\left(T_{s \|}+2 T_{s \perp}\right) / 3$ is the average temperature and $R_{s t}$ represents the transition rate from charge state $s$ to state $t$ caused by photoionization, collisional ionization, recombination, charge exchange. The Coulomb frequency $v_{s t}$ is defined as

$v_{s t}=\frac{1}{3} \frac{n_{t} m_{t}}{m_{s}+m_{t}}\left(\frac{2 \pi k T_{s t}}{\mu_{s t}}\right)^{-3 / 2} \frac{e_{s}^{2} e_{t}^{2}}{\epsilon_{0}^{2} \mu_{s t}^{2}} \ln \lambda$,

where $\epsilon_{0}$ is the permittivity of vacuum and $\ln \lambda$ accounts for the electric field's Debye screening.

The last term in (11) and the second to last term in (12) are higher-order, frictional heating terms that are not part of the collision terms derived by Janse et al. (2005). These terms have been added for collisions between helium ions and protons in the corona to improve energy conservation. In order to greatly simplify the derivation, as well as to obtain numerically tractable terms, the collision terms of Janse et al. (2005), as well as the collision terms of, e.g., Chodura \& Pohl (1971) and Demars \& Schunk (1979), were developed only up to first order in the ratio of the relative flow speed of the particles to the thermal speed. Since frictional heating is of second order in this ratio, it is thus not accounted for in the formal derivation of these equations. Being a second order effect, the frictional heating between protons and $\alpha$-particles is negligible in the low corona where flow speeds are small. It is also negligible in the solar wind where flow speed differences can be large, because collisions are negligible in this region. Hence only in a relative small region, where collisions are still important but the friction is sufficiently small to allow relative flow speeds that are not negligible compared to the thermal speed, may this term be important for the energy budget. Being confined to a small region, this term is therefore not essential for the total energy budget of the corona and solar wind. However, to improve the overall energy conservation of our calculations, which facilitates the interpretation of our results, we have nevertheless included a frictional heating term.

The formally correct frictional heating term is of course obtained by developing the collision term up to second order in the relative flow speed to thermal speed ratio. Whereas this is straightforward for a simple drifting Maxwellian velocity distribution (see, e.g. Schunk 1977), the collision integrals become exceedingly complex for gyrotropic velocity distributions, for which reason this has not been done in any of the available gyrotropic formulations. Given that this term is not essential for the solar corona, and will at most provide a modest improvement to the energy conservation, this formally correct approach is therefore neither worthwhile nor feasible. We have therefore adopted the much simpler approach of using the drifting Maxwellian expression for frictional heating (see Schunk 1977), and simply assumed that the thermal energy generated is split evenly between the three (perpendicular and parallel) degrees of freedom. Since the term is important only in a small region where highly skewed velocity distributions have not yet developed, using the drifting Maxwellian assumption in the collision integrals does not seem inappropriate. Since frictional heating between electrons and ions is much smaller than between ions (because of the small mass ratio), frictional heating is not included in the electron energy equations. With the frictional term included, the relative difference between the applied energy flux (heating) and radiation and solar wind energy losses is of order $1 \%$.

The last terms in (10) and (12) are introduced to mimic the two-stream instability in the outer solar wind, forcing protons and helium ions to flow at drift velocities that do not deviate by more than the local Alfvén speed $v_{\mathrm{a}}$. The collision frequency $v_{s t}^{2 \mathrm{str}}$ is nonzero when $\left|u_{s}-u_{t}\right|>v_{\mathrm{a}}$ and is given as

$$
\begin{aligned}
v_{s t}^{2 s t r} & =\frac{v_{\max }}{2}\left(\tanh \left(\frac{\Delta u_{0}}{\Delta u}\right)+\tanh \left(\frac{\Delta v_{\mathrm{a}}-\Delta u_{0}}{\Delta u}\right)\right) . \\
v_{\max } & =\frac{u_{\text {flow }}}{r_{2 \operatorname{str}}\left(1+\frac{\rho_{s}}{\rho_{t}}\right)} \\
\Delta v_{\mathrm{a}} & =\frac{\left|u_{s}-u_{t}\right|}{v_{\mathrm{a}}}
\end{aligned}
$$

Here $u_{s}$ and $u_{t}$ are the proton and ion flow speed, $v_{\mathrm{a}}=B / \sqrt{\mu_{0} \rho_{0}}$ is the local Alfvén speed, $\mu_{0}$ is the permeability of vacuum and $\rho_{0}$ is the total mass density. $\tanh \left(\Delta u_{0} / \Delta u\right)$ ensures that the collision frequency indeed is zero when the difference in drift speed is very small compared with the Alfvén speed. We have chosen $\Delta u_{0}=1.0$ and $\Delta u=0.2$. In a background flowing at $u_{\text {flow }}=300 \mathrm{~km} \mathrm{~s}^{-1}$ the flow speeds should couple roughly over a distance of $r_{2 \mathrm{str}}=3 R_{\mathrm{S}}$ when the proton-ion flow speed difference is significantly larger than the local Alfvén speed.

Apart from the frictional heating terms and the reactive terms (proportional to $R_{s t}$ ), the energy collision terms (11) and (12) are identical to those of Chodura \& Pohl (1971) and Demars \& Schunk (1979).

The radiative loss is included in the electron energy equation. The loss from excitation of neutral hydrogen (Ly $\alpha$ radiation) is calculated using the actual neutral hydrogen density obtained by the code. In addition, radiative loss from metallic ions is included assuming ionization equilibrium for these.

\section{Solar wind model}

The model starts in the chromosphere and goes out to $1 \mathrm{AU}$. We solve the equation set (1)-(5) for six particle species; namely neutral hydrogen, protons, and all charge states of helium, and electrons. The requirements of charge neutrality and no current replace the continuity and momentum equations for electrons, while the electric field is obtained from the electron momentum equation. The lower boundary is set in the chromosphere to assure that the boundary conditions are unaffected when we change the corona heating. In a radiation dominated model, where the radiation loss constitutes a substantial part of the energy flux, the transition region pressure is directly proportional to the downward heat flux from the corona. Thus a large downward heat flux gives a high transition region pressure, a higher corona density and a slower more massive solar wind. Starting at a temperature of less than $10^{4} \mathrm{~K}$, instead of somewhere in the transition region, also allows us to keep track of radiation loss and energy needed to bring the particles up to the corona.

At the lower boundary the total hydrogen density and the total helium density are set to $n_{\mathrm{H}}=10^{20} \mathrm{~m}^{-3}$ and $n_{\mathrm{He}}=$ $5 \times 10^{18} \mathrm{~m}^{-3}$. The outer boundary conditions are given by linear extrapolation, except that inward heat flow is not allowed for. The plasma is produced dynamically through photoionization of the neutral gas and collisional ionization in the transition 


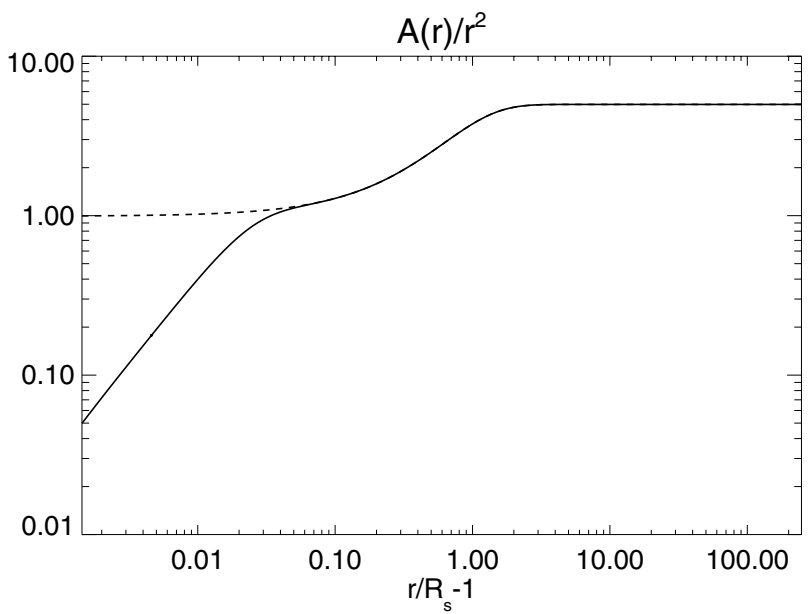

Fig. 1. Hole (dashed) and funnel (solid line) flux tube area divided by the heliocentric distance squared as a function of the distance.

region and lost through radiative and three-body recombination, contained in the $R_{s t}$ terms of (9)-(13).

The magnetic field structure is taken from Kopp \& Holzer (1976), with a flux tube area $A(r) \propto 1 / B(r)$ specified as

$A=A_{0}\left(\frac{r}{1 R_{s}}\right)^{2} F_{1} \cdot F_{2}$

where

$$
\begin{aligned}
& F_{1}=\frac{f_{\mathrm{m} 1} \mathrm{e}^{\left(r-r_{g 1}\right) / \sigma_{g 1}}+f_{g 1}}{\mathrm{e}^{\left(r-r_{g 1}\right) / \sigma_{g 1}}+1} \\
& F_{2}=\frac{f_{\mathrm{m} 2} \mathrm{e}^{\left(r-r_{g 2}\right) / \sigma_{g 2}}+f_{g 2}}{\mathrm{e}^{\left(r-r_{g 2}\right) / \sigma_{g 2}}+1} .
\end{aligned}
$$

Here $A_{0}$ is the area of the flux tube at the lower boundary and $R_{\mathrm{S}}$ is the solar radius and $f_{g 1}=1-\left(f_{\mathrm{m} 1}-1\right) \mathrm{e}^{\left(R_{\mathrm{S}}-r_{g 1}\right) / \sigma_{g 1}}$ (and similarly for $\left.f_{g 2}\right)$. Here $f_{\mathrm{m} 1} f_{\mathrm{m} 2}$ specifies how many times more the flux tube opens than a radial tube where $A=A_{0}\left(\frac{r}{1 R_{\mathrm{S}}}\right)^{2}, r_{g 1}$ indicates where the funnel expansion takes place and $r_{g 2}$ where the coronal hole superradial expansion occurs, while $\sigma_{g 1(2)}$ specify over what distances the expansions occur.

We will study two magnetic field structures: corona holes with and without a funnel (a flux tube narrowing) at the base. The "funnel" magnetic field structure undergoes two expansions, where $f_{m 1}=20, r_{g 1}=1.005 R_{\mathrm{S}}$ and $\sigma_{g 1}=0.01 R_{\mathrm{S}}$ and $f_{m 2}=5$, $r_{g 2}=1.3 R_{\mathrm{S}}$ and $\sigma_{g 2}=0.5 R_{\mathrm{S}}$. The parameters with subscript 1 represents the funnels main expansion close to the inner boundary, while the latter expansion is the same expansion as in the coronal hole model of Munro \& Jackson (1977). (In the funnel models of Li (2002); Esser et al. (2005) the first expansion varies from $f_{m 1}=13$ to 62 while the second expansion has a $f_{\mathrm{m} 2} \sim 7$.) The coronal hole without a funnel has the same values for $f_{m 2}, r_{g 2}$, and $\sigma_{g 2}$ as the funnel model, while $f_{m 1}=1$ (implying $F_{1}=1$ ). To facilitate comparison of the two geometries we let the funnel and hole models have the same flux tube area at $1 \mathrm{AU}$. We therefore choose $A_{0}=1 \mathrm{~m}^{2}$ in the hole model and $A_{0}=1 / 20 \mathrm{~m}^{2}$ in the funnel model. The expansion of the flux tubes are shown in Fig. 1. We note that apart from the funnel expansion at $r \lesssim 1.03 R_{\mathrm{S}}$, the two flow geometries are identical.

Energy is inserted into the chromosphere to balance radiation loss and in the corona to drive the solar wind. In the chromosphere electrons receive all heating (as the electrons here are thermally coupled to hydrogen and helium, it does not matter which particles we heat), choosing

$Q_{\| e m}^{\mathrm{CH}}=Q_{\perp e m}^{\mathrm{CH}}=\frac{2}{3} C_{\mathrm{e}} n_{\mathrm{e}} n_{\mathrm{H}}$,

where $C_{\mathrm{e}}=5 \times 10^{-39} \mathrm{~W} \mathrm{~m}^{3}$, e refers to electrons, and $n_{\mathrm{H}}$ is the sum of the neutral hydrogen and proton densities. The heating maintains a chromosphere temperature of $7000-8000 \mathrm{~K}$. Further up in the atmosphere this heating term is negligible since it is proportional to density squared.

In accordance with observations (Kohl et al. 1997) indicating high ion temperatures, most of the corona energy supply is given to protons and $\alpha$-particles. For both species the corona heating is deposited perpendicular to the magnetic field, to reproduce the coronal temperature anisotropy $T_{\perp}>T_{\|}$observed by Kohl et al. (1997) and Frazin et al. (2003). The ion corona heating rates are

$Q_{m s \|}=0$

and

$Q_{m s \perp}=-\frac{1}{A} \frac{\mathrm{d} F_{m s}}{\mathrm{~d} r}$

where $F_{m s}$ is the applied energy flux and $s=p, \alpha$. The energy flux is exponentially damped and starts at the heliocentric distance $r_{1 i}$,

$F_{m s}=F_{m s 0}$ for $r<r_{1 i}$

$F_{m s}=F_{m s 0} \exp \left(-\frac{r-r_{1 i}}{H_{m i}}\right)$ for $r \geq r_{1 i}$.

Here $F_{m s 0}$ is the supplied energy flux at the base of the model and $H_{m i}$, which will be varied, is the damping length which determines over how large region the heating is inserted. The heating is started at $r_{1 i}=1.03 R_{\mathrm{S}}$. A total applied energy flux $F_{m i 0}=390 \mathrm{~W}$ is deposited in the proton and $\alpha$-particles, while the distribution between protons and $\alpha$-particles will be varied.

The electrons are heated isotropically in the corona,

$Q_{m e \|}=Q_{m e \perp}=-\frac{2}{3} \frac{1}{A} \frac{\mathrm{d} F_{m e}}{\mathrm{~d} r}$

where $F_{m e}$ is given by (26)-(27). The electron heating rate parameters are $F_{m e 0}=40 \mathrm{~W}, r_{1 e}=1 R_{\mathrm{S}}$ and $H_{m e}=0.5 R_{\mathrm{S}}$. Thus approximately $10 \%$ of the total coronal energy input of $430 \mathrm{~W}$ goes into electrons.

The model has an adaptive grid to handle the transition region, which moves in response to changes in the downward heat flux. The grid density $\rho_{z}$, the number of grid points per unit distance $z$, is proportional to the electron temperature gradient and given by (see Dorfi \& Drury 1987)

$\rho_{z}(z) \propto \sqrt{1+\left(\frac{z_{\mathrm{av}}}{T_{e, \mathrm{av}}} \frac{\mathrm{d} T_{\mathrm{e}}}{\mathrm{d} z}\right)^{2}}$,

where "av" denotes an appropriate average of the electron temperature $T_{\mathrm{e}}$ and $z$, the distance from the lower boundary. The grid density varies with time, but the total number of points remains unchanged at 480 points. A grid density equation is solved together with the five transport equations (1)-(5) for each particle species. A partly implicit finite difference scheme is used to solve these coupled equations. The equations are discretized and integrated forward in time from a initial solution until a steady state solution is reached. 
When $u_{s} \approx \sqrt{k T_{s \|} / m_{s}}$ a singularity in the equations (as well as in the equations of Demars \& Schunk 1979) may cause the drift speed and parallel temperature to oscillate, resulting in an instability. To avoid this the hydrogen and helium heat flux are damped at $1.8 R_{\mathrm{S}}$. At this location the temperature has already peaked and the heat flux is positive (i.e., flowing outwards). The outward heat flux is smaller than the other energy flux terms, and damping it merely means that the wind accelerates slightly faster than it would otherwise, but it does not affect the asymptotic wind speed far from the Sun.

In the collisionless outer solar wind the equation set produces a constant parallel temperature and a perpendicular temperature which decreases as $r^{-2}$. Thus in the outer solar wind the equations give a parallel temperature much larger than the perpendicular temperature, which does not agree with observations. Therefore, when $T_{\|}>2 T_{\perp}$, which is the onset condition for the firehose instability, a coupling between $T_{\|}$and $T_{\perp}$ is introduced which lowers $T_{\|}$and increases $T_{\perp}$ (ensuring energy conservation).

Since frictional drag from hydrogen is insufficient to pull helium out of the chromosphere in the hole model, we introduce an artificial outward force on neutral helium $f_{\text {artificial }}=-\frac{3}{4} m_{\mathrm{He}} G$, which increases the scale height of helium to that of hydrogen when the chromospheric flow speed is small. In the funnel model no such force is applied.

\section{Numerical results}

Before we consider outflow originating in a funnel, we present, for comparison, a solar wind from the coronal hole flow tube of Fig. 1 without a funnel at the base. We start the model from a hydrogen-proton-electron solar wind solution where $5 \%$ helium has been added in the entire solution. To produce a fast solar wind, energy is inserted in the outer corona, with an ion damping length $H_{m i}=0.9 R_{\mathrm{S}}$, and $20 \%$ of the ion heating is given to the $\alpha$-particles (this is the observed fraction of the solar wind energy flux carried by $\alpha$-particles). The equations are integrated forward in time until a steady state has been reached, shown in Fig. 2.

The chromospheric hydrogen and helium densities, summed over charge states, decease rapidly with distance, with a density scale height close to the hydrogen hydrostatic equilibrium scale height at $\sim 7000 \mathrm{~K}$. In the transition region, located at $r=1.004 R_{\mathrm{S}}$, the pressure is constant and the density is inversely proportional to the temperature. In the outer solar wind, where the flow speed is constant, the density is inversely proportional to the distance squared (flux conservation).

The helium abundance, the helium-to-hydrogen particle number ratio, remains approximately at $5 \%$ in the chromosphere, where the artificial force we introduced sets the helium scale height equal to the hydrogen scale height so that the helium abundance stays approximately constant throughout the chromosphere. In the lower transition region, $T \sim 10^{4}-10^{5} \mathrm{~K}$, the helium abundance falls to $1 \%$ as helium is accelerated by the thermal force. In the corona there is a helium buildup around $r=1.1 R_{\mathrm{S}}$ to $19 \%$ before the ratio falls to $5 \%$ in the outer solar wind.

The proton flow speed increases with distance and becomes supersonic at $r \approx 1.1 R_{\mathrm{S}}$, reaching a terminal speed of nearly $900 \mathrm{~km} \mathrm{~s}^{-1}$. The proton and $\alpha$-particle speeds are almost identical, except in the region with helium buildup where the protons flow faster and in the transition region where helium flows five times faster than hydrogen. The model yields a hydrogen flux density at $1 \mathrm{AU}$ of $\left(n_{\mathrm{H}} u_{\mathrm{H}}\right)_{\mathrm{E}}=1.5 \times 10^{12} \mathrm{~m}^{-2} \mathrm{~s}^{-1}$ and a helium flux density of $\left(n_{\mathrm{He}} u_{\mathrm{He}}\right)_{\mathrm{E}}=7.5 \times 10^{10} \mathrm{~m}^{-2} \mathrm{~s}^{-1}$, which are about $20 \%$ less than the observed fast solar wind.

In the chromosphere the model has a nearly constant temperature of $7300 \mathrm{~K}$. The temperature increases rapidly in the thin transition region. In the corona the electron temperature peaks at $8.5 \times 10^{5} \mathrm{~K}$, the protons reach $1.1 \times 10^{7} \mathrm{~K}$, and the $\alpha$-particles reach $5.4 \times 10^{7} \mathrm{~K}$. The temperatures decouple in the lower corona at $r \approx 1.03 R_{\mathrm{S}}$, which is where the ion heating begins. At this location the proton and $\alpha$-particle flow speeds also decouple. This shows that the coronal densities are so low that the collisional coupling between species is small, even in the very low corona. Such high proton and $\alpha$-particle temperatures are necessary if a high-speed wind is to be driven by coronal heating only.

Of the $430 \mathrm{~W}$ deposited in the flow tube, about $20 \mathrm{~W}$ is transported downwards, mostly by electron heat conduction. The protons conduct less than $5 \mathrm{~W}$ downwards and the $\alpha$-particles less than $1 \mathrm{~W}$, despite that most of the energy is deposited in the protons and $\alpha$-particles. Note also that while the electron heat flux changes sign at the $T_{\mathrm{e}}$ maximum, indicating "classical" collision-dominated heat conduction where $q_{\mathrm{e}} \propto-\mathrm{d} T_{\mathrm{e}} / \mathrm{d} r$, the proton and $\alpha$-particle heat fluxes are positive well below the temperature maximum, showing that they do not behave like a collision-dominated gas in the corona.

The radiation loss of the transition region above $10^{4} \mathrm{~K}$ is found by integrating the radiative loss per volume per second, $f_{\text {rad }}$, from $r=r_{\mathrm{T}}$ where $T_{\mathrm{e}}=10^{4} \mathrm{~K}$ and out to $r$,

$F_{\mathrm{rad}}(r)=\int_{r_{\mathrm{T}}}^{r} f_{\mathrm{rad}}\left(r^{\prime}\right) A\left(r^{\prime}\right) \mathrm{d} r^{\prime}$.

The total radiation loss of the transition region (most of which occurs at $T=1-4 \times 10^{5} \mathrm{~K}$ ) is only about $7 \mathrm{~W}$, which means that most of the downward heat flux is not converted to radiation, but rather used to heat the upstreaming plasma (enthalpy flux) (see Sect. 4.4).

The hole model produces a fast solar wind solution, with a helium abundance of 5\% except in a region in the corona with up to $20 \%$. The helium buildup is not large enough to significantly alter the background of hydrogen, protons and electrons, which is very similar to the corresponding hydrogen-proton-electron solution without helium.

Even though new transport equations reduce the thermal force on $\alpha$-particles by approximately a factor of 3, compared with, e.g., the equations used by Lie-Svendsen et al. (2003), the helium abundance in the corona is still high, approximately $20 \%$. This shows that the coronal helium abundance is not sensitive to the magnitude of the thermal force. Rather, it is the energy balance of the system that affects the coronal helium abundance. This is in contrast to closed coronal loops, where the thermal force has a large impact on coronal abundances (see Killie et al. 2005).

\subsection{Coronal hole with a funnel at the base}

We then turn to the funnel model, using the same coronal heating parameters as above. The numerical model is started from the hole solution, and integrated until a steady state has been achieved. In Fig. 3 the funnel solution and the hole solution of Fig. 2 are shown. The funnel model has a slightly flatter density curve in the region $r \approx 1.004-1.03 R_{\mathrm{S}}$ where the funnel flux tube undergoes its large expansion, elsewhere the density curves are very similar. The funnel helium abundance increases slightly in the chromosphere and only falls to $3 \%$ in the transition region, showing that the thermal force has less influence in the funnel 

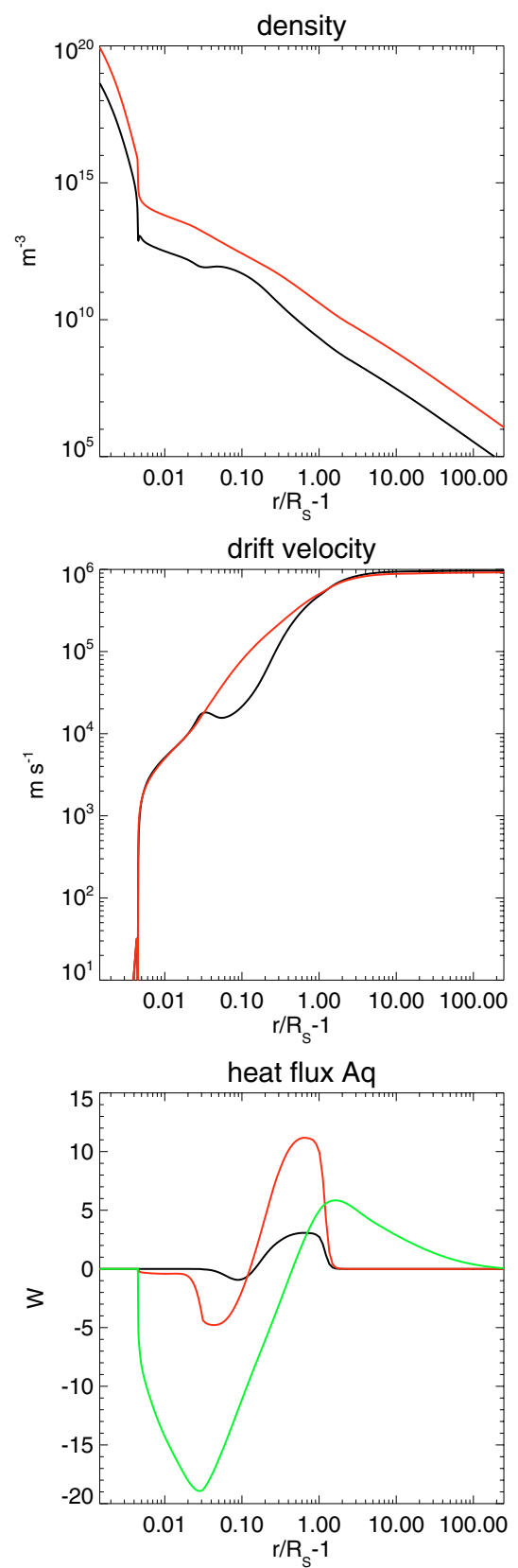
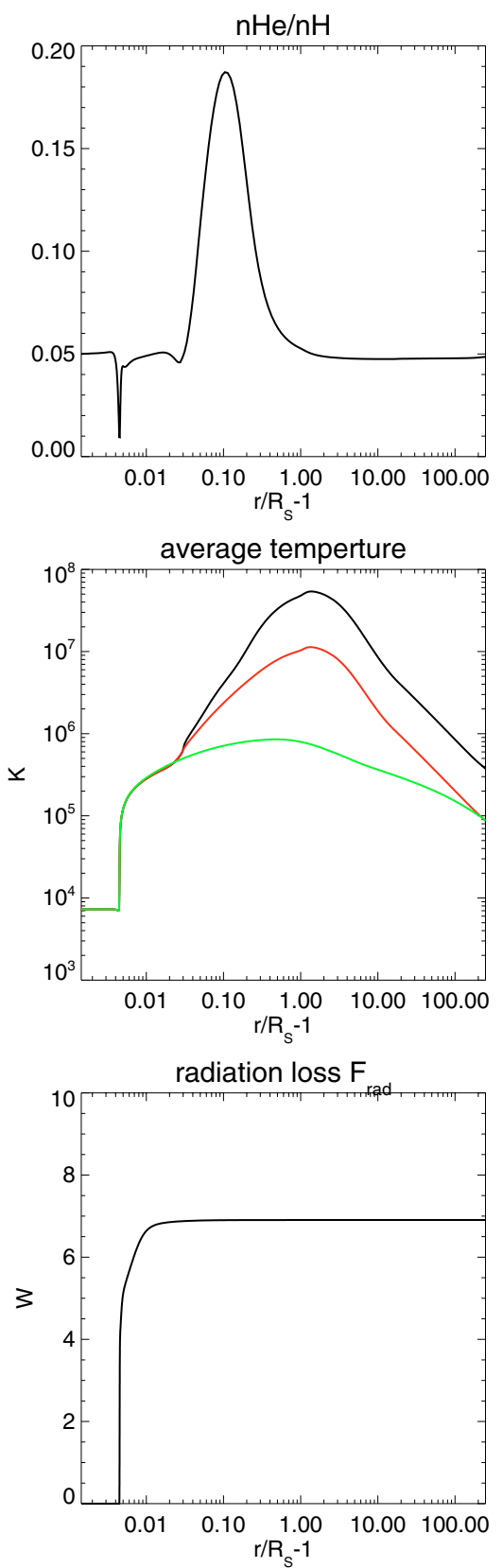

Fig. 2. Solutions for the hole model with a damping length $H_{m i}=0.9 R_{\mathrm{S}}$. In the density plot black represents helium and red hydrogen; both densities are summed over charge states. The helium abundance is $n_{\mathrm{He}} / n_{\mathrm{H}} \equiv\left(n_{\mathrm{He}}+n_{\mathrm{He}^{+}}+\right.$ $\left.n_{\mathrm{He}^{++}}\right) /\left(n_{\mathrm{H}}+n_{\mathrm{p}}\right)$. In the drift speed, temperature and heat flux plots red corresponds to protons, black is $\alpha$-particles and green is electrons. The radiation loss is defined in (30) as the energy radiated above the $T_{\mathrm{e}}=10^{4} \mathrm{~K}$ temperature level. model. In the corona the helium abundance peaks at $19 \%$ for the corona hole and $14 \%$ for the funnel model. Further out the ratio decreases to $5 \%$ in both models.

In the funnel model the hydrogen particle flux density at 1 AU is $1.5 \times 10^{12} \mathrm{~m}^{-2} \mathrm{~s}^{-1}$, as in the hole model, and the helium particle flux density is $6.8 \times 10^{10} \mathrm{~m}^{-2} \mathrm{~s}^{-1}$, deviating less than $10 \%$ from the hole model. Therefore, in the chromosphere the funnel particles flow approximately 20 times faster than the hole particles. When the funnel expands the speed remains nearly constant at $\sim 25 \mathrm{~km} \mathrm{~s}^{-1}$ instead of increasing as in the hole model. After the funnel expansion is done the flow speeds in the two models are almost identical.

The temperature panel shows that in the funnel model the transition region is located at slightly lower altitude and the ion and electron temperatures decouple further down, due to faster flow speeds. The higher electron temperature in the funnel, in the lower corona, is a consequence of the flux tube geometry; a higher temperature is required to conduct a given heat flux into a contracting tube. The downward heat flux in the funnel model is almost the same as in the hole model, with minimums of $16 \mathrm{~W}$ and $19 \mathrm{~W}$, respectively. The radiation loss in the funnel is even smaller than in the hole, with only $2 \mathrm{~W}$ of the $\sim 16 \mathrm{~W}$ conducted down converted to radiation compared with $7 \mathrm{~W}$ in the hole. Hence almost all of the downward heat flux is used to heat the plasma from chromospheric to coronal temperatures.

We have thus produced a "reasonable" solar wind from a corona hole with a funnel at the base. In the introduction we argued that in a funnel it might be difficult to conduct sufficient heat into the transition region in order to sustain the observed solar mass flux, because the solar wind energy loss might prevent the electron temperature from reaching the required (higher) value. We note from Fig. 3 that the energy conducted down is only slightly less in the funnel than in the hole, and that only in the very low corona is the funnel electron temperature higher, while the maximum electron temperature is almost identical in the two geometries, and the solar wind energy loss is therefore 

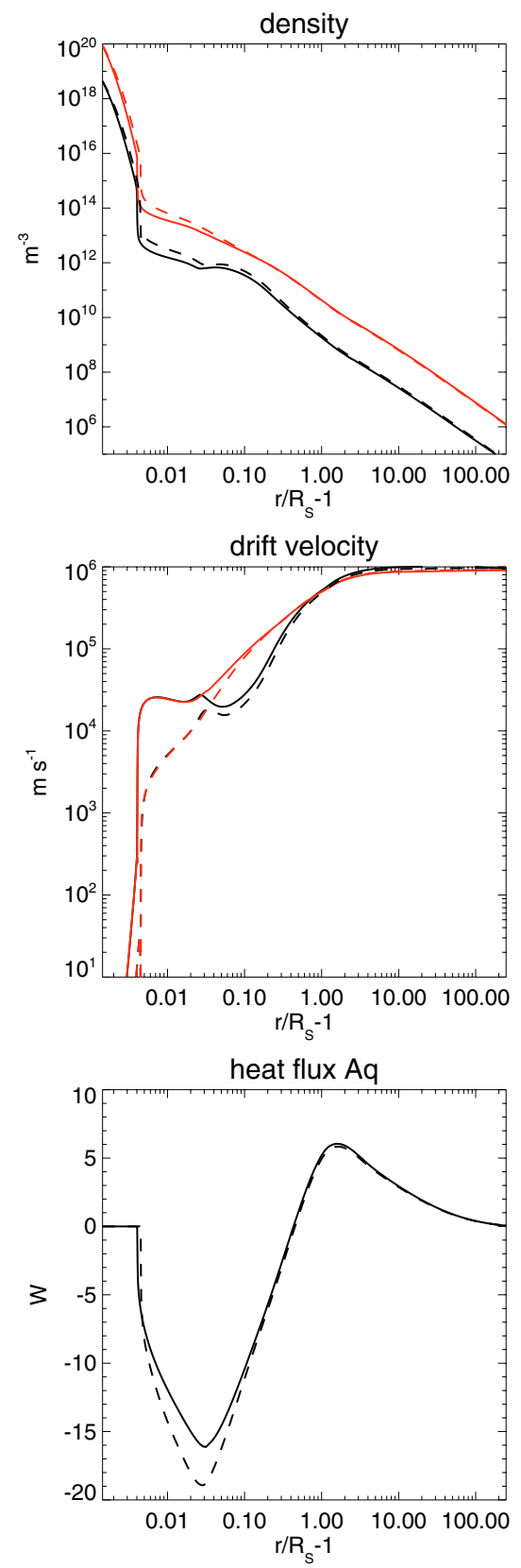
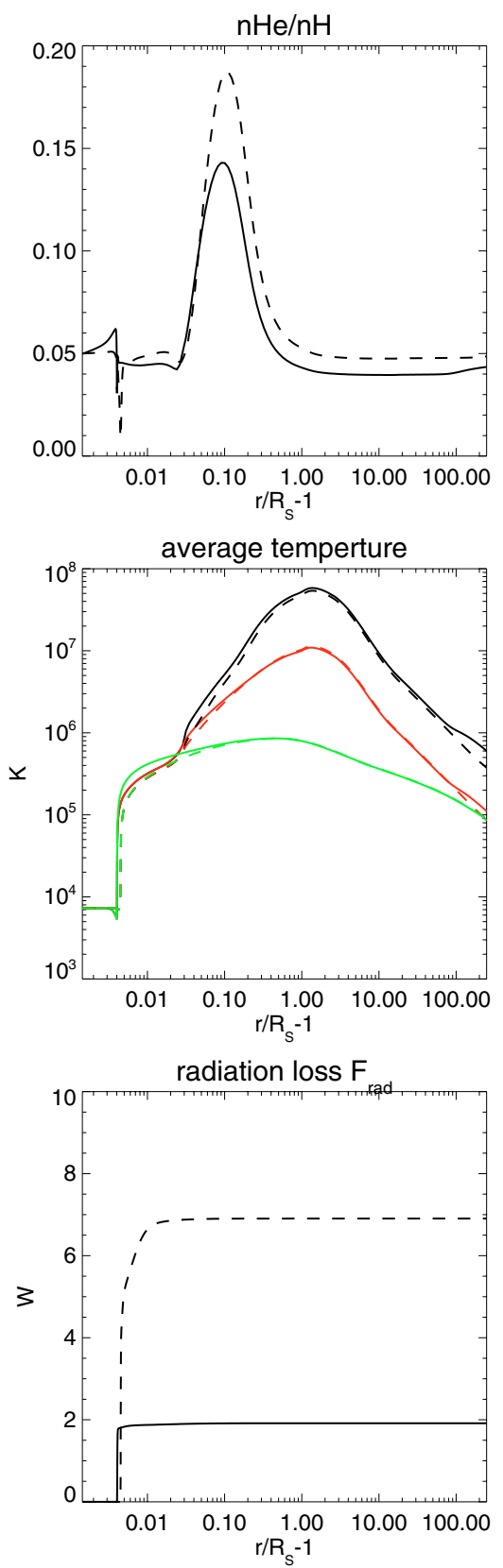

Fig. 3. Comparison of the hole (dashed) and the funnel (solid line) solutions with $H_{m i}=$ $0.9 R_{\mathrm{S}}$. The hole solution and the notation are the same as in Fig. 2. unaffected. Most of the downward heat flux is provided by protons and $\alpha$-particles, but in a low density corona (as in this solution) the collisional transfer of energy to the electrons only happens in the very low corona, well below the electron temperature maximum. When electrons receive energy very close to the Sun, little of this energy will be lost in the solar wind, and therefore we are ensured that this energy will be conducted into the transition region sustaining the required mass flux, even in a funnel geometry. However, the question still remains whether an even narrower funnel (i.e., expanding much more than a factor of 20) can provide the solar wind mass flux. We return to this question in Sect. 5.

Similarly, we note that the other concern raised in the introduction, that the radiative loss from a funnel would lead to a coronal pressure that is much too high to provide a high-speed wind, does not occur in this particular solution. Although the heat flux density is approximately a factor 15 higher in the funnel transition region, almost all of this energy is used to heat the upstreaming plasma, and the radiative loss therefore is so small that the pressure of the transition region and corona remains very low.

The hole and funnel models produce very similar solutions, which only differ in the region where the funnel has its large flux tube expansion. For both the corona and the solar wind it seems irrelevant whether the magnetic field geometry contains a funnel or not at the coronal base. The main effect of the funnel is that we no longer have to introduce an artificial force in the chromosphere to prevent helium from settling, and that the helium abundance at $1 \mathrm{AU}$, where helium and hydrogen flow at the same speed, is set far down in the chromosphere (in our model at the lower boundary), as discussed in the introduction.

We find that both models produce wind speeds and particle fluxes in good agreement with observations. However, the modeled coronal electron densities are approximately a factor 10 smaller than the densities inferred from observations (Koutchmy 1977; Munro \& Jackson 1977; Fisher \& Guhathakurta 1995; Doschek et al. 1997; Wilhelm et al. 1998). In this kind of model, where radiation is accounted for, coronal 
densities can only be changed by changing the form of coronal heating. Hence there is no simple way of increasing the coronal densities without affecting the whole solar wind solution. Hansteen et al. (1997) and Lie-Svendsen et al. (2003), who did not consider funnel geometries, found that models with higher coronal densities also had higher helium abundances in the corona.

\subsection{Heating closer to the Sun}

To try to increase the coronal densities we move the coronal heating further down, by decreasing the ion damping length from $H_{m i}=0.9 R_{\mathrm{S}}$ to $H_{m i}=0.4 R_{\mathrm{S}}$. The electron heating and the total heating remain unchanged. By heating further down in the corona, we expect more energy to be conducted down and the radiation loss to increase, which will result in a higher transition region pressure and thus a higher coronal density.

Figure 4 shows that the decrease in damping length does not significantly alter the solutions. The hole and funnel still yield very similar solutions, except where the funnel expands. At $1 \mathrm{AU} u_{\mathrm{p}}=820 \mathrm{~km} \mathrm{~s}^{-1}$ and $u_{\alpha}=860 \mathrm{~km} \mathrm{~s}^{-1}$ in the hole, while $u_{\mathrm{p}}=940 \mathrm{~km} \mathrm{~s}^{-1}$ and $u_{\alpha}=980 \mathrm{~km} \mathrm{~s}^{-1}$ in the funnel. The funnel hydrogen and helium flux densities have not changed from the $H_{m i}=0.9 R_{\mathrm{S}}$ solutions in Fig. 3, while the hole hydrogen and helium fluxes at $1 \mathrm{AU}$ have increased slightly from $1.5 \times 10^{12} \mathrm{~m}^{-2} \mathrm{~s}^{-1}$ and $7.5 \times 10^{10} \mathrm{~m}^{-2} \mathrm{~s}^{-1}$ in the $H_{m i}=0.9 R_{\mathrm{S}}$ case to $1.7 \times 10^{12} \mathrm{~m}^{-2} \mathrm{~s}^{-1}$ and $8.8 \times 10^{10} \mathrm{~m}^{-2} \mathrm{~s}^{-1}$. The maximum helium abundance has decreased to $7 \%$ in the funnel, implying that the proton and $\alpha$-particle flow speeds are close everywhere.

With a smaller damping length for the ion coronal heating, which still starts at $r=1.03 R_{\mathrm{S}}$, the proton and $\alpha$-particle temperature gradients in the inner corona have steepened. The maximum ion temperatures have increased by $30 \%$, peaking closer to the solar surface compared with the solution with $H_{m i}=0.9 R_{\mathrm{S}}$. The electron temperature only undergoes minor changes.

In the hole the steepened proton temperature gradient has increased the maximum downward proton heat flux from $5 \mathrm{~W}$ with $H_{m i}=0.9 R_{\mathrm{S}}$ to $8 \mathrm{~W}$ with $H_{m i}=0.4 R_{\mathrm{S}}$. However, in the funnel model protons conduct down less energy; the maximum proton heat flux has decreased slightly from $5 \mathrm{~W}$ to $4 \mathrm{~W}$. Despite the steepened proton temperature gradient, the downward proton heat flux in the funnel model has not increased, implying that it is not proportional to the temperature gradient here, demonstrating that the protons do not behave like a collision-dominated gas in the inner corona. Inserting more energy into the ions in the inner corona does not increase the downward electron heat flux, showing that the collisional energy transfer to electrons is small. In the funnel, electrons actually conduct down slightly less energy than in the $H_{m i}=0.9 R_{\mathrm{S}}$ case. Thus, the radiation loss is smaller, and the densities are even lower.

If we further decrease the ion damping length to $H_{m i}=$ $0.2 R_{\mathrm{S}}$, the solutions (not shown) are not altered noticeably; we still have a fast solar wind with low coronal densities. Hence, heating mostly protons and $\alpha$-particles, even very close to the Sun, does not yield coronal densities that agree with observations.

\subsection{Reducing the $\alpha$-particle heating}

We try another approach to increase the coronal densities by rearranging the partition of ion coronal heating while the damping length is kept constant at $H_{m i}=0.4 R_{\mathrm{S}}$. This also allows us to explore the consequence of the locking of the helium flux to the hydrogen flux in the funnel. Since the helium particle flux is $5 \%$ of the hydrogen flux, and helium is four times heavier than hydrogen, helium needs $20 \%$ of the energy to escape out of the gravitational field and achieve the same asymptotic flow speed as protons. Helium is continuously being brought up to the corona and when the helium heating is reduced to the point where helium does not receive enough energy to escape the gravitational field, helium will build up in the corona, causing an increase in the electron density as well. We reduce the heating of $\alpha$-particles from $20 \%$ of the $F_{m i 0}=390 \mathrm{~W}$ applied ion energy flux to $10 \%$. The solution with $20 \%$ helium heating is used as the initial solution. We further reduce the heating of $\alpha$-particles from $10 \%$ to $5 \%$, starting from the $10 \%$ solution. The funnel solutions with $20 \%, 10 \%$ and $5 \%$ helium heating are shown in Fig. 5.

Decreasing the helium heating from $20 \%$ to $10 \%$ does not produce large changes; the electron density increases less than $20 \%$ and the solar wind particle fluxes are unchanged. The $\alpha$-particle flow speed is reduced in the inner region, $r<30 R_{\mathrm{S}}$, further out the $\alpha$-particle and proton flow speeds are coupled by the two-stream instability term (17)-(19), which accelerates $\alpha$-particles at the expense of protons. With $10 \%$ of the heating, $\alpha$-particles still have enough energy to escape the gravitational potential and do not need to reduce the helium particle flux. Thus, the hydrogen particle flux does not have to be reduced, either, in order to accommodate the reduced energy input in helium.

The solution with $5 \%$ helium heating, on the other hand, is extremely different from the solutions with $10 \%$ and $20 \%$ helium heating, and is dominated by a huge helium buildup in the corona and far into the solar wind. The energy flux deposited in helium is no longer sufficient to lift the original helium particle flux out of the gravitational field, and the helium abundance in the corona increases. The frictional force from $\alpha$-particles on protons will then reduce the proton flux, and hence also the helium flux, until energy balance once again is established. Thus the particle flux densities are now lower, $\left(n_{\mathrm{H}} u_{\mathrm{H}}\right)_{\mathrm{E}}=8.9 \times 10^{11} \mathrm{~m}^{-2} \mathrm{~s}^{-1}$ and $\left(n_{\mathrm{He}} u_{\mathrm{He}}\right)_{\mathrm{E}}=3.7 \times 10^{10} \mathrm{~m}^{-2} \mathrm{~s}^{-1}$, and the inner coronal electron density is 50-150 times larger than with $20 \%$ helium heating. The coronal electron density is now considerably higher than observations indicate; at $1.05 R_{\mathrm{S}}$ the electron density is $5 \times 10^{14} \mathrm{~m}^{-3}$, while observations (Wilhelm et al. 1998) indicate $n_{\mathrm{e}}=7 \times 10^{13} \mathrm{~m}^{-3}$.

The helium abundance peaks at 15 at $r=1.5 R_{\mathrm{S}}$. In this region the $\alpha$-particles have a flow speed of less than $1 \mathrm{~km} \mathrm{~s}^{-1}$ and the $\alpha$ temperature is actually lower than the proton temperature. The $\alpha$-particle temperature has decreased drastically from the temperature obtained with $20 \%$ helium heating and now peaks at $5 \times 10^{6} \mathrm{~K}$, while the coronal electron temperature is almost doubled. The ion and electron temperatures remain coupled until $r=1.2 R_{\mathrm{S}}$, caused by the high densities.

In a region with high helium abundance, and high electron temperature $\left(T_{\alpha \|} / T_{\mathrm{e} \|}<2\right)$, the electric force acting on protons is larger than gravity. Leer et al. (1992) found that if the helium abundance is smaller than $25 \%$, the protons and electrons are not significantly affected by the helium in the background. But as the helium abundance become larger than 25\%, the solution changes character and protons undergo a rapid acceleration. Solutions with a helium abundance of $25 \%$ or higher will be referred to as "helium dominated". The solution with $5 \%$ helium heating has a helium abundance larger than $25 \%$ in a rather large region, $r \approx 1.04-10 R_{\mathrm{S}}$. The electric field accelerates the protons from a few hundred $\mathrm{m} \mathrm{s}^{-1}$ at $r=1.04 R_{\mathrm{S}}$ to $900 \mathrm{~km} \mathrm{~s}^{-1}$ over a distance $\sim 1 R_{\mathrm{S}}$. In the same region the $\alpha$-particle flow speed is less than $10 \mathrm{~km} \mathrm{~s}^{-1}$. 

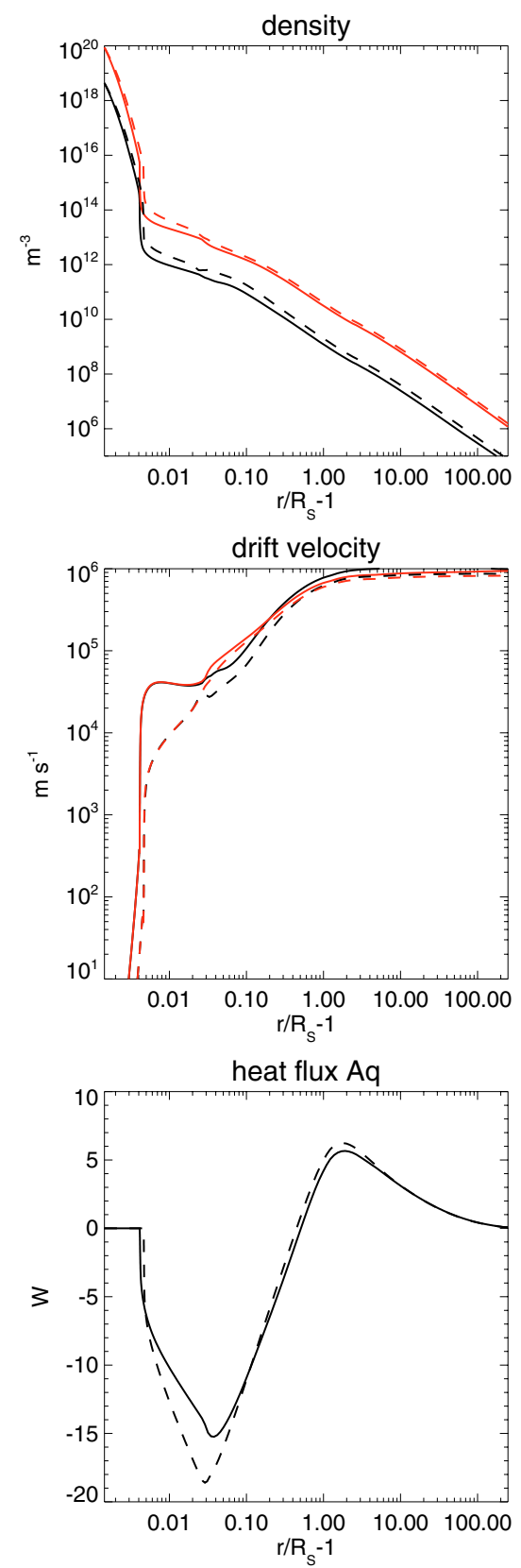
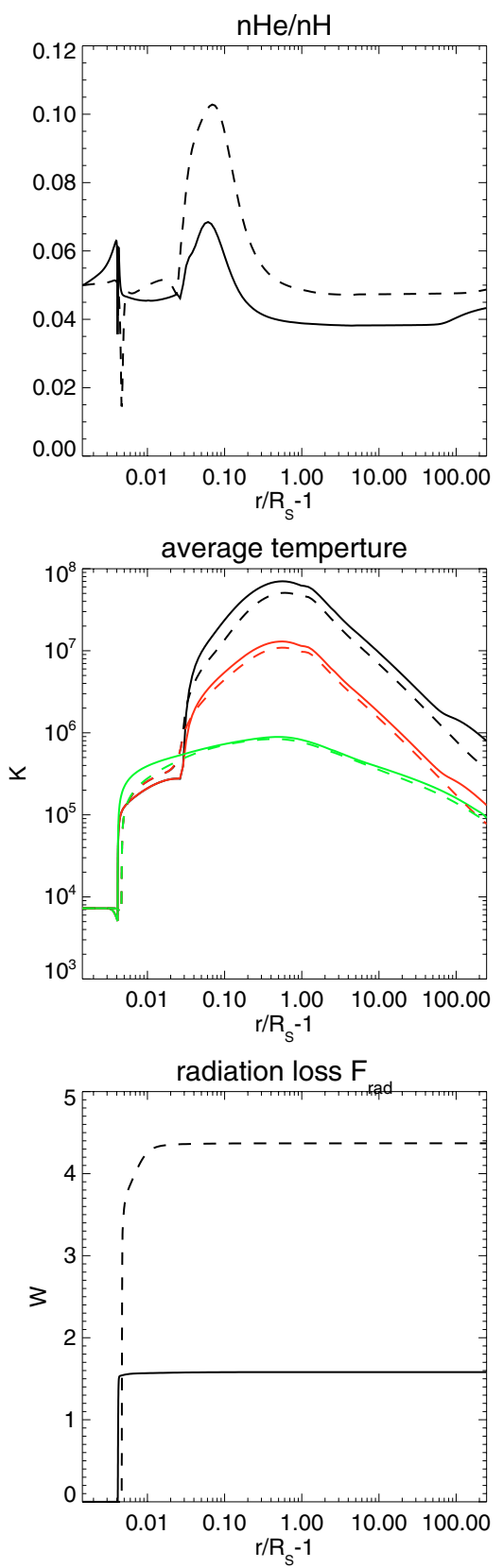

Fig. 4. Comparison of the hole (dashed) and the funnel (solid line) solutions with $H_{m i}=0.4 R_{\mathrm{S}}$, using the same color coding as in Fig. 2.
Compared with the model with $20 \%$ helium heating, $5 \%$ helium heating leads to a 10 times larger downward electron heat flux and a much higher electron temperature and lower lying transition region, consistent with the increased heat flux. The radiation loss has increased from less than $2 \mathrm{~W}$ to about $165 \mathrm{~W}$. In the model with 5\% helium heating, energy is inserted into a region which is collision-dominated, and more than $1 / 3$ of the energy is conducted down and radiated away. By comparison, in the $10 \%$ and $20 \%$ solutions the downward conducted energy is mainly used to heat the upstreaming plasma, and they have tiny radiation losses of less than $2 \mathrm{~W}$.

The hole solutions (not shown) qualitatively undergo the same changes as the funnel solutions when the $\alpha$-particle heating is reduced, but the changes are not as huge. The $5 \%$ helium heating solution has an (inner) coronal electron density 10-25 times larger than the $20 \%$ helium heating density, compared with a 50-150 time increase in the funnel. The decrease in helium heating from $20 \%$ to $5 \%$ lowers the particle fluxes by $35 \%$ and increases the maximum helium abundance from $10 \%$ to $500 \%$.
The maximum downward heat flux increases five times and almost $80 \mathrm{~W}$ is radiated away.

By decreasing the helium heating we managed to increase the coronal electron density, but the increase is too large, compared with observations, especially in the funnel model. The solutions have a huge helium buildup in the corona and the $\alpha$ particle temperatures are very low. The large downward heat flux density is now converted mostly into radiation, and the pressure of the transition region and corona therefore becomes very high. Because the heat flux density becomes much higher in the funnel than without the funnel at the base (for the same total heat flux $A q_{\mathrm{e}}$ ), we can understand why the funnel solution undergoes much larger changes than the hole solution when the helium heating is reduced. In the funnel solution with $5 \%$ helium heating the problem anticipated in the introduction - that a very high heat flux density in the funnel needs to be converted into radiation, thus leading to a coronal density that is much too high - has certainly materialized. 

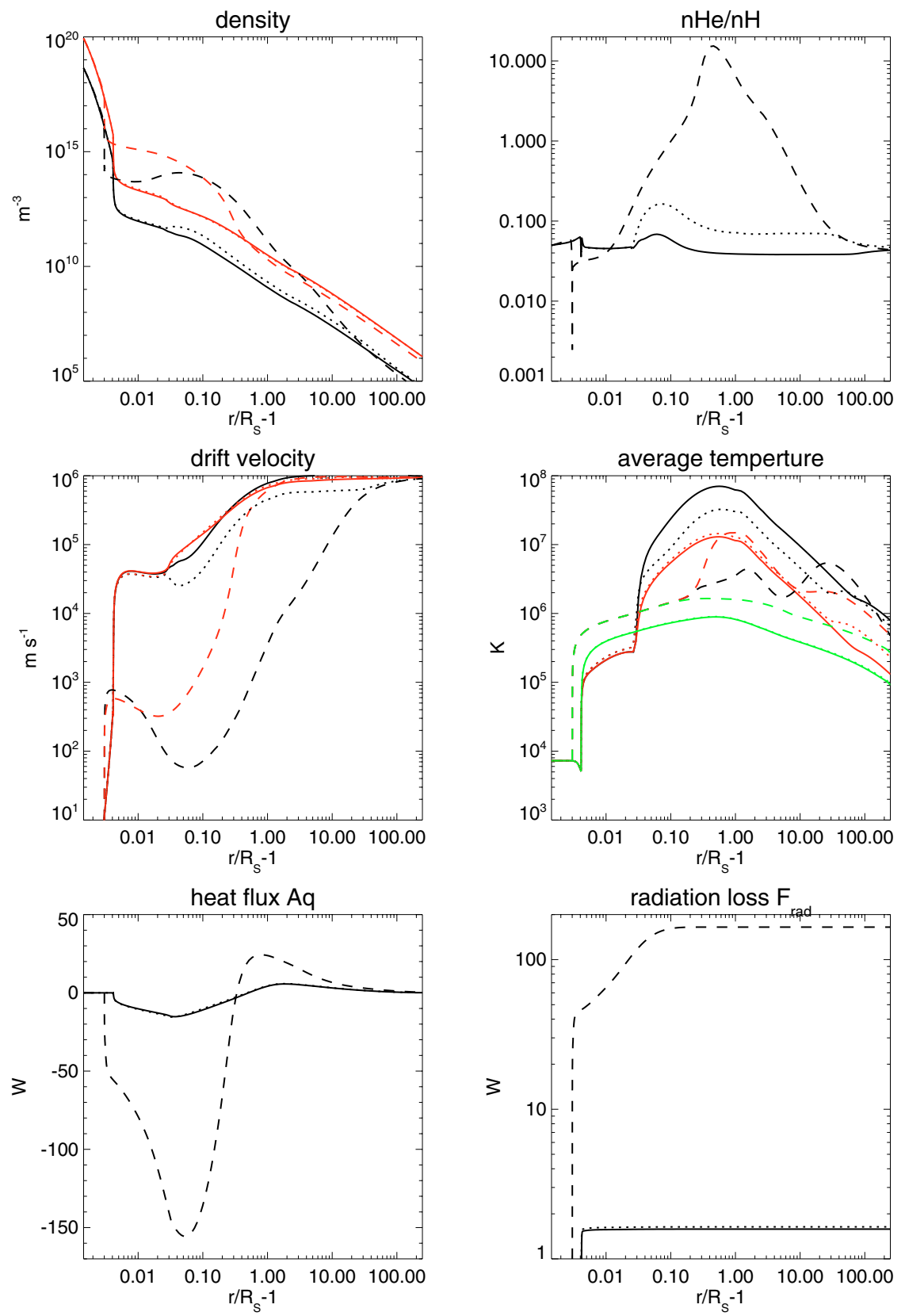

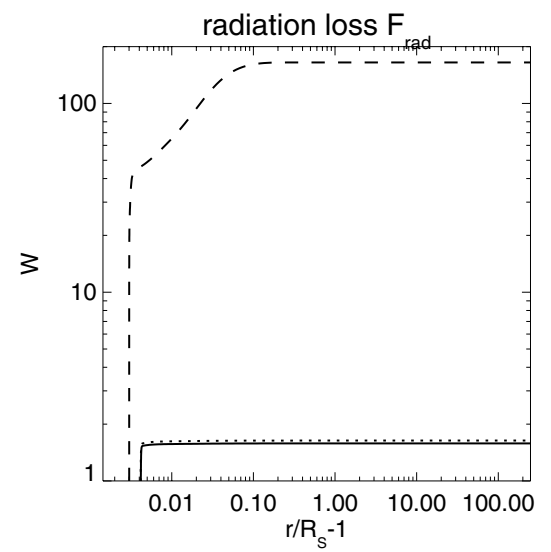

Fig. 5. Comparison of funnel solutions with $H_{m i}=0.4 R_{\mathrm{S}}$ and $5 \%$ helium heating (dashed), $10 \%$ helium heating (dotted) and 20\% helium heating (solid line). The notation is the same as in Fig. 2.
If helium does not receive enough energy to escape the gravitational potential, it builds up in the corona, and the solution undergoes huge changes. Hence supplying helium with adequate heating is essential in a solar wind emerging from a funnel.

\subsection{Two solar wind modes}

In the model with $5 \%$ helium heating we found a very high coronal density. In this case energy is inserted in a region which is collision-dominated, and the $\alpha$-particle temperature reaches only $\sim 5 \times 10^{6} \mathrm{~K}$. The coronal densities are so high, and the collisional coupling between species in the corona so strong, that one may question whether increasing the $\alpha$-particle heating back to, say, the original $20 \%$ will cause the solution to revert to the high-speed solution of Fig. 4 if we start from the 5\% solution in Fig. 5. Or will the collisional coupling remain high so that the extra energy deposited in $\alpha$-particles is transferred to protons and electrons instead of increasing the $\alpha$-particle temperature? In the latter case we would also expect the helium abundance to remain high.

Figure 6 shows the result of increasing the helium heating back to the original $20 \%$, but starting from the $5 \%$ solution of Fig. 5 and integrating until a steady state is reached. The solution indeed does not revert to the low density solution of Fig. 4, showing that the same model input parameters, particularly the same heating parameters and geometry, can have two very different solutions, depending on where the model was started. We arrive at a solution which resembles the slow solar wind at Earth, while the original solution had properties at $1 \mathrm{AU}$ similar to the fast solar wind. In the following, the two solutions in Fig. 6 will be referred to as the slow and fast wind solutions.

At $1 \mathrm{AU}$ the slow wind solution has flow speeds $u_{\mathrm{p}}=$ $470 \mathrm{~km} \mathrm{~s}^{-1}$ and $u_{\alpha}=450 \mathrm{~km} \mathrm{~s}^{-1}$, and hydrogen and helium particle flux densities of $2.2 \times 10^{12} \mathrm{~m}^{-2} \mathrm{~s}^{-1}$ and $1.0 \times 10^{11} \mathrm{~m}^{-2} \mathrm{~s}^{-1}$, respectively. The fast wind has flow speeds at $1 \mathrm{AU} u_{\mathrm{p}}=$ $940 \mathrm{~km} \mathrm{~s}^{-1}$ and $u_{\alpha}=980 \mathrm{~km} \mathrm{~s}^{-1}$, and a hydrogen flux density of $1.5 \times 10^{12} \mathrm{~m}^{-2} \mathrm{~s}^{-1}$ and helium flux density $6.8 \times 10^{10} \mathrm{~m}^{-2} \mathrm{~s}^{-1}$. The 

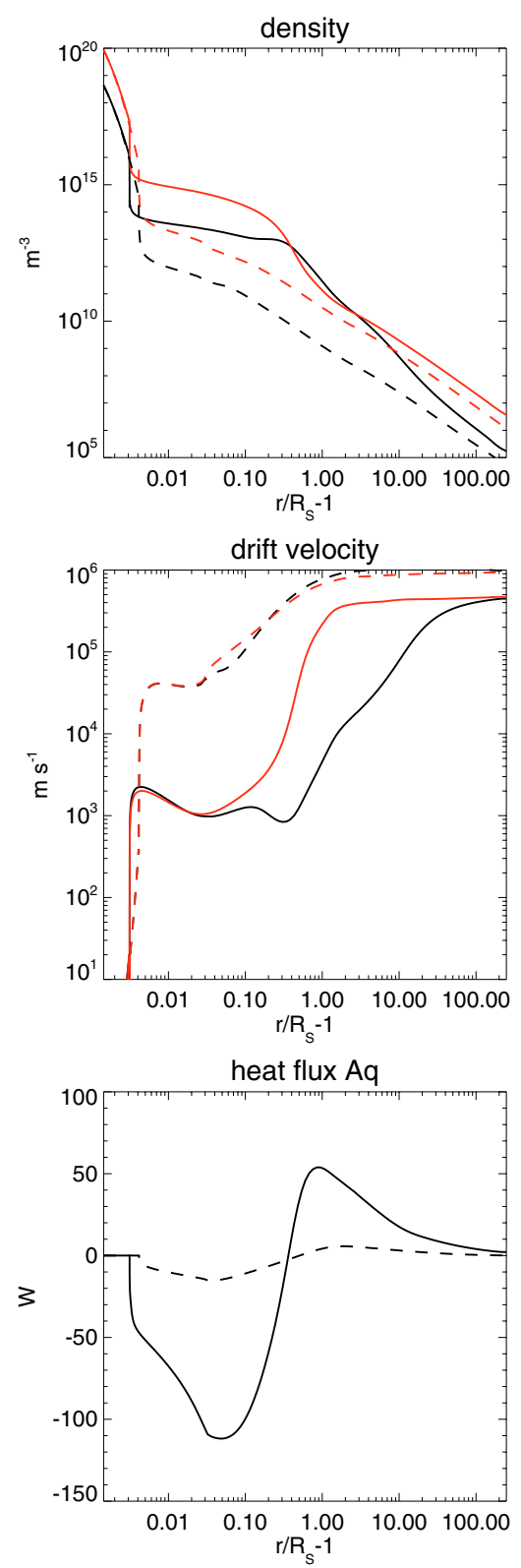
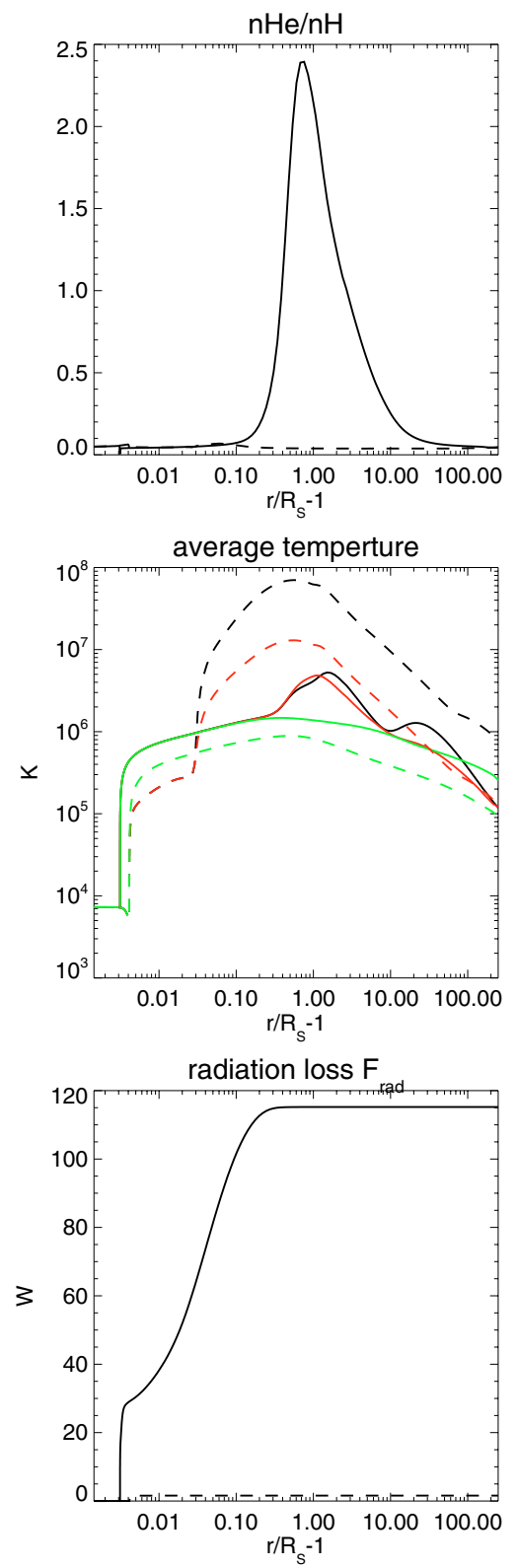

Fig. 6. Comparison of funnel solutions with $H_{m i}=$ $0.4 R_{\mathrm{S}}$ and $20 \%$ helium heating. Solutions with $5 \%$ helium heating are used as initial solution for the slow wind solution (solid line). The fast wind solution (dashed) is the same solution as in Fig. 4. slow wind has electron densities in the inner corona that are up to 120 times higher than the densities in the fast wind solution. In the fast solution the helium abundance is $4-7 \%$. The slow wind helium abundance peaks at $240 \%$ at $r=1.8 R_{\mathrm{S}}$ and it is larger than $25 \%$, corresponding to a helium-dominated solution, in a rather large region $r=1.2-11 R_{\mathrm{S}}$.

When we decreased the helium heating from $20 \%$ of the ion coronal heating to $5 \%$, the particle fluxes decreased. As the helium heating is returned to the original $20 \%$, the particle fluxes are higher than the original fluxes, showing that the increase in downward heat flux and corresponding higher coronal density, which favors a higher mass flux, more than offsets the frictional drag on hydrogen caused by the higher helium abundance.

The energy flux contributions for the fast and slow solar wind solutions in Fig. 6 are presented in Fig. 7. The enthalpy flux is given as

$$
F_{\text {enthalpy }}=A \sum_{s} n_{s} u_{s}\left(\frac{3}{2} k T_{s \|}+k T_{s \perp}\right) \text {, }
$$

where the sum extends over electrons and all charge states of hydrogen and helium. The total heat flux is a sum of all the individual heat fluxes

$F_{\text {heat }}=A \sum_{s} q_{s}$

The kinetic energy is

$F_{\text {kinetic }}=A \sum_{s} n_{s} u_{s} \frac{1}{2} m_{s} u_{s}^{2}$

and the gravitational potential energy flux is

$F_{\text {gravity }}=A G M_{\mathrm{S}}\left(\frac{1}{R_{\mathrm{S}}}-\frac{1}{r}\right) \sum_{s} n_{s} u_{s} m_{s}$,

where $M_{\mathrm{S}}$ is the solar mass, and we have chosen the potential to be zero at the solar surface. The applied energy flux is the sum of the fluxes of the form (26)-(27) for protons, $\alpha$-particles, and electrons. 

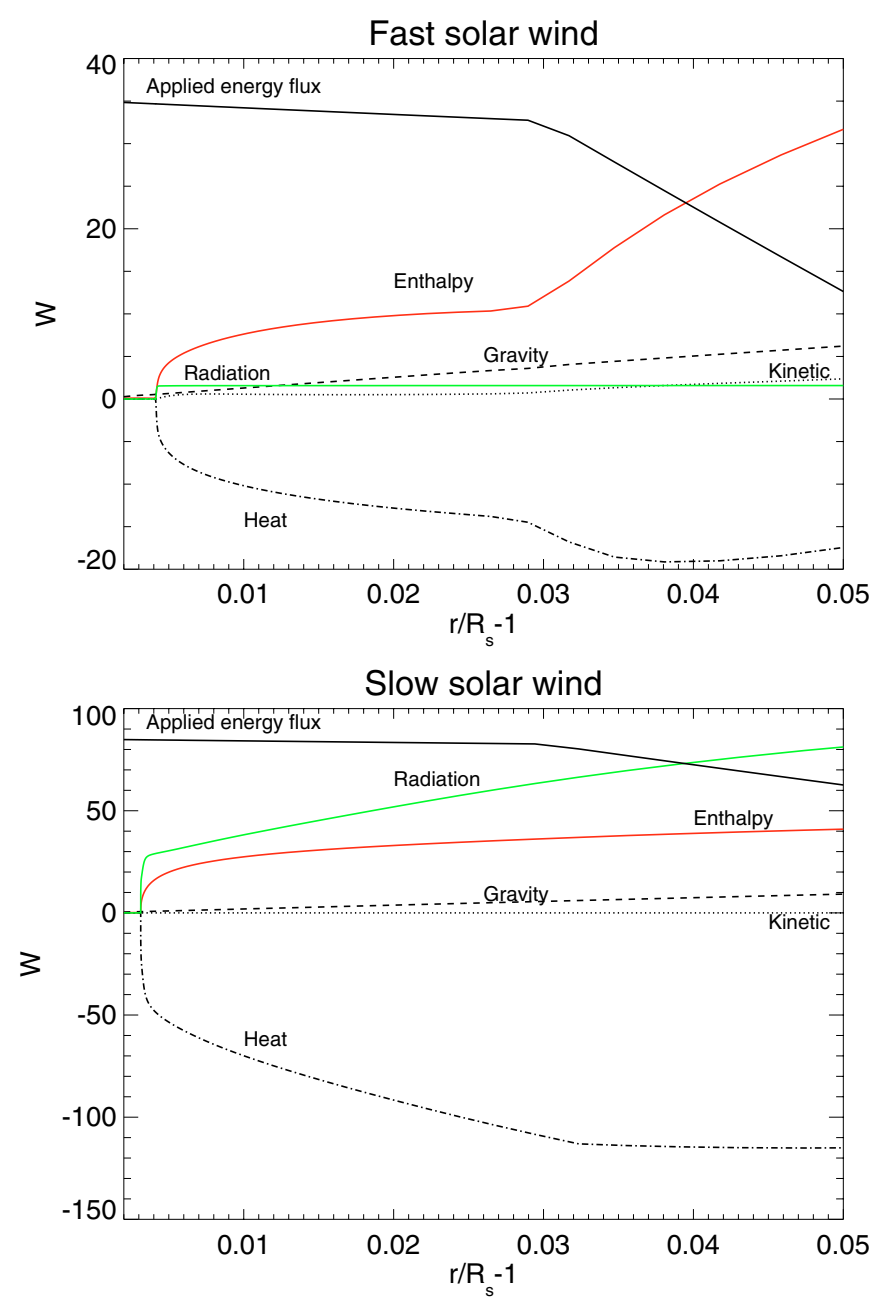

Fig. 7. Energy fluxes in the lower atmosphere for the two funnel solutions with $H_{m i}=0.4 R_{\mathrm{S}}$ and $20 \%$ helium heating shown in Fig. 6. To make the fluxes comparable $395 \mathrm{~W}(345 \mathrm{~W})$ has been subtracted from the applied energy heating flux (26)-(27) in the fast wind (slow wind). The radiation loss is defined in (30). The enthalpy, heat, flux and the gravitational energy flux are defined in (31)-(34).

In the fast solar wind solution the energy flux budget in the transition region and the lower corona is enthalpy dominated: Of the $16 \mathrm{~W}$ conducted down at $r=1.03 R_{\mathrm{S}}$, approximately $12 \mathrm{~W}$ is converted into enthalpy flux, heating of the upstreaming gas, about $4 \mathrm{~W}$ goes into gravitational potential energy, only $2 \mathrm{~W}$ goes into radiation, while the kinetic energy flux is less than $1 \mathrm{~W}$. The slow solar wind on the other hand is radiation dominated: At $r=1.03 R_{\mathrm{S}}$ the downward energy flux is $109 \mathrm{~W}$, of this $64 \mathrm{~W}$ is radiated away, $36 \mathrm{~W}$ is used as enthalpy flux, $6 \mathrm{~W}$ goes into gravitational potential energy, and the kinetic energy flux is negligible. (The $2 \mathrm{~W}$ reduction in the applied energy flux from the lower boundary and up to $r=1.03 R_{\mathrm{S}}$ is caused by electron heating which starts at the lower boundary.) In the slow solution the coronal densities are so high that there is significant radiative loss fairly high in the corona; between $r=1.05 R_{\mathrm{S}}$ and $1.2 R_{\mathrm{S}}$ (corresponding to electron temperatures of $1.1 \times 10^{6} \mathrm{~K}$ and $1.4 \times$ $10^{6} \mathrm{~K}$, respectively) another $40 \mathrm{~W}$ is lost as radiation.

The very different energy flux budget for the fast and slow wind can be used to understand the difference in the solutions: the higher downward heat flux in the slow wind means that there is ample energy to sustain an increased enthalpy flux, and thus an increased solar wind mass flux. Moreover, the higher coronal
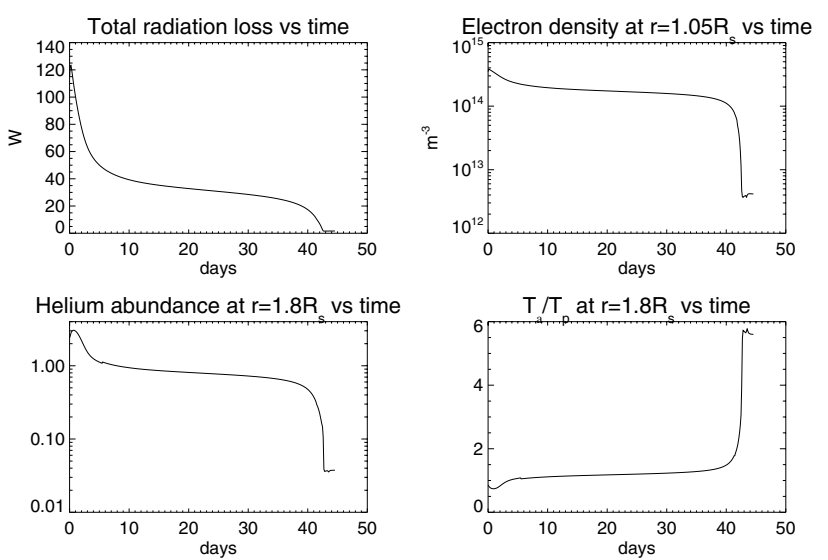

Fig. 8. Time development of the funnel model with $H_{m i}=0.5 R_{\mathrm{S}}$, where the slow solar wind in Fig. 6 is used as starting point.

density, associated with the large downward heat flux density, typically leads to a higher mass flux and thus a slower terminal wind speed.

While we have two solutions in the funnel geometry, the hole model returns to the "original" solution (the same solution as in Fig. 4) when the helium heating is increased from 5\% back to $20 \%$. The hole geometry does not produce co-existing solutions.

The two co-existing solutions have the same heating parameters and geometry, but they have different initial solutions. Withbroe (1988) argued that heating and geometry were enough to determine the solution, but we find that the starting solution also has to be specified for the funnel geometry to have a unique solution. We believe this to be the first case where heating parameters and geometry do not uniquely determine the solar wind solution.

\section{Discussion}

Given the existence of the two modes, how can the Sun make the transition between them? And how "robust" are they - that is, is only a small, temporary perturbation in some parameter sufficient to cause the switch? Or do the two modes only exist in a very narrow parameter regime, making it unlikely that they can be realized on the Sun? After all, from the polar coronal holes we always observe the high-speed wind, which indicates that in these regions, at least, such co-existing modes probably do not exist. Similarly, what are the requirements for two co-existing modes - is the funnel really necessary? Is helium necessary? These are some of the issues we address below.

We find two co-existing solutions for ion damping lengths in the range $H_{m i}=0.2-0.45 R_{\mathrm{S}}$. As the damping length is increased to $0.5 R_{\mathrm{S}}$ and beyond, the fast solar wind is the only possible solution. Hence the two modes are not confined to a narrow range of proton and $\alpha$-particle heating parameters, although the existence of the high-density mode does seem to require that a large fraction of the solar wind energy is deposited close to the Sun.

To shed some light on how the system can make the transition from the slow to the fast mode, we have run the model with $H_{m i}=0.5 R_{\mathrm{S}}$, starting from the slow $H_{m i}=0.4 R_{\mathrm{S}}$ solution in Fig. 6. Figure 8 shows the time development of the total radiation loss, the electron density, the helium abundance and the average temperature ratio $T_{\alpha} / T_{\mathrm{p}}$ at a given distance $r$. The total radiation loss falls rapidly in the beginning, decreasing from $115 \mathrm{~W}$ to $50 \mathrm{~W}$ in about 5 days. The radiation loss continues to 
decrease, but then at a much slower rate. After 40 days the loss undergoes a large change as it falls from $15 \mathrm{~W}$ to less than $2 \mathrm{~W}$ in less than 2 days.

When the model starts the electron density in the inner corona, at $r=1.05 R_{\mathrm{S}}$, is $3.5 \times 10^{14} \mathrm{~m}^{-3}$. The density falls to $2 \times 10^{14} \mathrm{~m}^{-3}$ within a few days and then decreases very slowly to $\sim 1 \times 10^{14} \mathrm{~m}^{-3}$ over the course of a month. After 40 days the density is reduced suddenly, within one day by a factor of 25 , to the steady state density $4 \times 10^{12} \mathrm{~m}^{-3}$. The helium abundance at $r=1.8 R_{\mathrm{S}}$, where the initial solution assumes the maximum helium abundance, undergoes the same time evolution as the radiation loss and the electron density. The helium abundance is rapidly reduced to $\sim 1$, then only undergoes small changes until 40 days have passed. At this point the helium abundance plummets to 0.05 and the solution is no longer helium dominated.

In the beginning the average proton temperature at $r=$ $1.08 R_{\mathrm{S}}$ is approximately equal to the average $\alpha$-particle temperature, and remains so for 40 days, showing that the collisional coupling persists for a very long time. As the other variables, the temperature ratio then undergoes a large change over only a few days, showing that the thermal coupling between protons and $\alpha$-particles suddenly ceases, allowing the $\alpha$-particles to become much hotter than protons and sufficiently hot to escape the gravitational potential.

What causes the slow wind to change into a fast wind? In the region $r<1.3 R_{\mathrm{S}}$ the ions and electrons had the same temperature and the slow wind was collision-dominated in the corona. The $\alpha$-particles gave away most of the energy inserted in them. As more heating is inserted further out in the corona, where there are fewer collisions, the electron heat flux decreases slightly as electrons receive less energy from the ions. Since the downward electron heat flux density is strongly connected to the radiation loss and the transition region pressure (and the coronal density), these also undergo reductions. The $\alpha$-particle temperature increases very slowly, as the $\alpha$-particles manage to retain more of the energy deposited in them.

Most of the $\alpha$-particle heating lost in collisions are given to protons, which transfer this heating along with the heating inserted directly in the protons to electrons. The energy transfer rate from $\alpha$-particles to protons is $\propto n_{\mathrm{p}}\left(T_{\alpha}-T_{\mathrm{p}}\right)\left(T_{\alpha}+4 T_{\mathrm{p}}\right)^{-3 / 2}$. As the proton density decreases, the temperature difference $T_{\alpha}-T_{\mathrm{p}}$ has to increase. Initially, $T_{\alpha}$ increases slowly, but when $T_{\alpha} \gtrsim 4 T_{\mathrm{p}}$ the increase in $T_{\alpha}-T_{\mathrm{p}}$ has to be more rapid. The energy transfer rate has a maximum at $T_{\alpha} / T_{\mathrm{p}}=11$, beyond which "runaway" heating would occur. However, the model never reaches this value because the solar wind energy loss becomes large well before this value is attained, and because $T_{\mathrm{p}}$ increases as the collisional transfer of energy to electrons is reduced. The $\alpha$ particles at $1.8 R_{\mathrm{S}}$ become hot enough to escape the gravitational potential when $T_{\alpha}>3.4 \times 10^{7} \mathrm{~K}\left(T_{\alpha}>2.9 \times 10^{7} \mathrm{~K}\right.$ when we take the electric force into account). The helium abundance falls below $25 \%$, at which point the protons and electrons are essentially no longer affected by helium. The thermal decoupling then allows for a fast wind solution.

While it takes more than a month for the corona and solar wind to switch mode when $H_{m i}$ is increased from $0.4 R_{\mathrm{S}}$ to $0.5 R_{\mathrm{S}}$, a much shorter time is needed when the start and end solutions are of the same mode, and therefore similar. For instance, if we start with the fast solution of Fig. 6, and increase $H_{m i}$ to $0.5 R_{\mathrm{S}}$, it takes only a few hours for the corona to make the small adjustments to the new steady state solution.

To check whether the solutions with $0.2 R_{\mathrm{S}}<H_{m i}<0.45 R_{\mathrm{S}}$ have reached steady states, we have run the slow wind models with $H_{m i}=0.4 R_{\mathrm{S}}$ and $0.45 R_{\mathrm{S}}$ for $t=4 \times 10^{7} \mathrm{~s}$ (more than 450 days). The solutions do not change.

The exact time evolution when the system switches mode will of course depend on the form of heating. We have chosen heating rates per unit volume which do not change over time. As the helium density in the corona is reduced when $H_{m i}$ is increased from 0.4 to $0.5 R_{\mathrm{S}}$, and we start from the slow solution in Fig. 6, the heating rate per $\alpha$-particle will increase. This will exacerbate the rapid temperature increase seen after 40 days in Fig. 8. Had we chosen instead, e.g., a constant heating rate per particle, we would expect a less rapid rise in temperature.

A different form of heating might also not produce two coexisting solutions. The two solutions in Fig. 6 have the same volume heating rate, while the heating rate per particle is very different. What we have shown is that, for a given applied energy flux deposited in the corona, the system can have two different solutions which have very different coronal densities and solar wind solutions, while the total energy flux in the solar wind does not deviate by more than about $25 \%$ between the two solutions (caused by the much higher radiative loss in the slow solution). This is also seen in the observed slow and fast solar wind, where the high flow speed of the fast wind is largely compensated for by the higher mass flux of the slow wind, making the fast wind energy flux only about $25 \%$ higher than the slow wind energy flux. Since we know from numerical modelling that most of the energy deposited in an open corona will end up in the solar wind (Hansteen \& Leer 1995), the similar energy fluxes in the two types of solar wind imply that the total energy flux deposited in the source regions of the fast and slow wind must be quite similar and may even be identical if the radiative loss is higher from the source region of the slow wind.

In Fig. 9 electron densities in the two solutions presented in Fig. 6 are compared with values inferred from observations of coronal holes, the source region of the fast solar wind. The slow wind mode has a coronal electron density 100 times larger than the density in the fast wind mode. While the electron density in the slow wind is larger than observed values, the fast wind density is too low. By varying the helium heating we are not able to produce a gradual transition in the coronal density, nor produce a high speed wind with coronal densities that agree with observations.

Is it possible to produce a gradually increasing coronal electron density in the fast wind by increasing the electron heating? We have kept $H_{m i}=0.4 R_{\mathrm{S}}$ and the $\alpha$-heating at $20 \%$, and increased the electron heating $F_{m e}$ (and thus the total energy deposition rate). When the electron heating is increased to $200 \mathrm{~W}, 5$ times the original electron heating, the model responds by gradually increasing the electron density in the corona. However, it is still too low compared with observations; at $1.05 R_{\mathrm{S}}$ we find $n_{\mathrm{e}}=2 \times 10^{13} \mathrm{~m}^{-3}$, while observations have $n_{\mathrm{e}} \approx 7 \times 10^{13} \mathrm{~m}^{-3}$. Moreover, the particle fluxes become too high for a fast wind solution, with $\left(n_{\mathrm{H}} u_{\mathrm{H}}\right)_{\mathrm{E}}=4.5 \times 10^{12} \mathrm{~m}^{-2} \mathrm{~s}^{-1}$ and $\left(n_{\mathrm{He}} u_{\mathrm{He}}\right)_{\mathrm{E}}=$ $2.2 \times 10^{11} \mathrm{~m}^{-2} \mathrm{~s}^{-1}$, more than twice the fast solar wind flux. There is no large helium buildup; the helium abundance has a maximum value of $10 \%$. The inner corona is still collisionless and almost all of the downward conducted energy is used to heat the upwelling plasma.

When the electron heating is further increased to $250 \mathrm{~W}$, there is a "jump" in the solution. The coronal electron density increases tenfold from $200 \mathrm{~W}$ to $250 \mathrm{~W}$ heating, and is more than twice the observed density. The hydrogen flux at $1 \mathrm{AU}$ is $\left(n_{\mathrm{H}} u_{\mathrm{H}}\right)_{\mathrm{E}}=5.7 \times 10^{12} \mathrm{~m}^{-2} \mathrm{~s}^{-1}$ and the helium flux is $2.8 \times 10^{11} \mathrm{~m}^{-2} \mathrm{~s}^{-1}$, about three times the observed fast solar wind fluxes. The helium abundance has a maximum of $14 \%$. The 


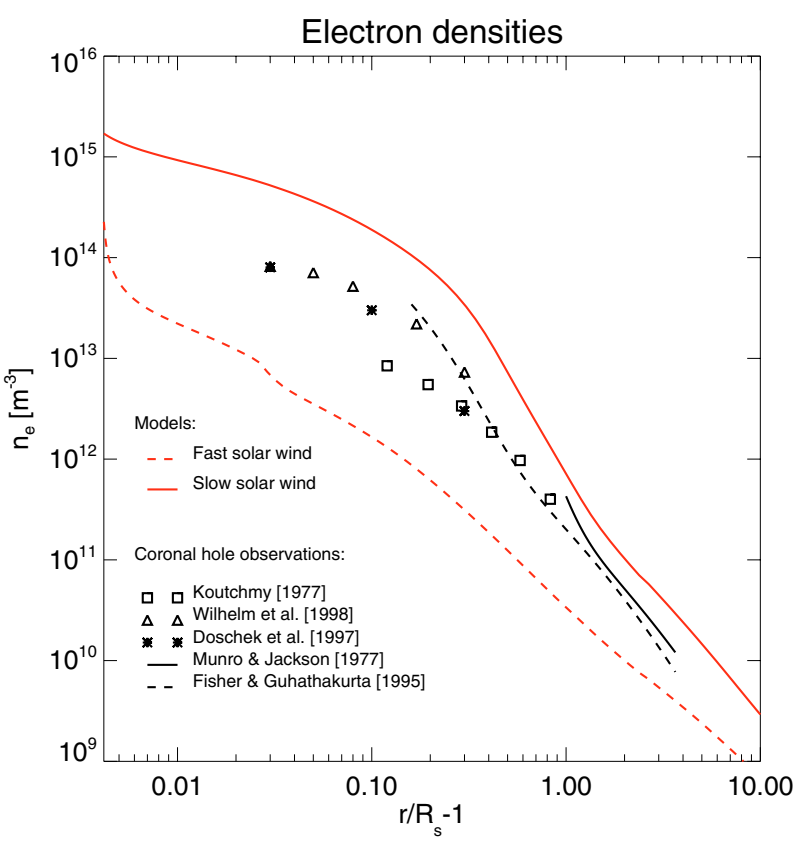

Fig. 9. Coronal electron densities from observations, the modeled electron densities from the fast and slow funnel solution in Fig. 6 .

inner coronal densities have become high enough to collisionally couple the ions and the electrons and the downward heat flux maximum has increased from $65 \mathrm{~W}$ to $131 \mathrm{~W}$. The radiation loss goes from $6 \mathrm{~W}$ to $51 \mathrm{~W}$, but most of the energy still goes into heating the vast amount of upwelling gas and the solution is enthalpy dominated. This result shows that it is possible to obtain a high-density corona that does not have a high (i.e., $\gtrsim 25 \%$ ) helium abundance. However, it has not been possible to produce a high-speed wind with a reasonable mass flux.

While the additional electron heating produces too high particle fluxes, decreasing the helium coronal heating (and keeping the total heating rate constant) results in a very large helium abundance. Either way we are not able to construct a fast solar wind with high coronal densities, and that has reasonable particle fluxes and is not helium-dominated in the corona.

If we start the model from the solution with $250 \mathrm{~W}$ electron heating, and reduce the electron heating back to the original $40 \mathrm{~W}$, the steady state solution is the slow wind mode presented in Fig. 6. Thus we have produced the same two co-existing modes, this time by varying the electron heating. The corona is collision-dominated and most of the ion heating is transferred to the electrons. Hence the maximum downward heat flux is still high, $131 \mathrm{~W}$ at the beginning and $112 \mathrm{~W}$ when the steady state is reached, despite that the additional electron heating is removed. However, since the radiative loss (below $1.03 R_{\mathrm{S}}$ ) is still about $60 \mathrm{~W}$, only $50 \mathrm{~W}$ is available to heat the upwelling gas, which is not large enough to sustain the initial particle flux. Hence the particle flux decreases and the helium abundance increases. As said before, a large helium abundance in the corona will afflict a large frictional force on the outflowing protons, decreasing the particle fluxes. Since the downward heat flux remains high and the particle fluxes are considerably reduced from the initial solution, by nearly a factor of 3 , the radiative loss remains high.

We have not been able to produce two co-existing solutions without helium, neither by varying the electron damping length $H_{m e}$ nor by increasing the electron heating and then reducing it. Moreover, without helium we do not find "discrete" transitions from one solution to another; the solution varies smoothly as the heating is varied.

For the case with $H_{m i}=0.4 R_{\mathrm{S}}$ we found that the hole model (which has $f_{m 1}=1$ ) only had one, enthalpy flux dominated and thus fast, solar wind solution. Reducing the funnel expansion $f_{m 1}$ from 20 to 5, while keeping the heating parameters unchanged, we find that the model still produces two distinctly different, coexisting solutions. As discussed in the introduction, if the funnel does not expand fast enough $\left(f_{m 1} \lesssim 5\right)$, the frictional force acting on neutral helium in the chromosphere from neutral hydrogen is not sufficient to lift helium up to the corona, the hydrogen and helium fluxes are no longer locked to each other, and the solar wind helium abundance becomes lower than 5\%.

Hence both a narrow funnel at the coronal base and the presence of helium seem to be necessary in order for the system to be able to switch between two very different, co-existing modes. When coronal densities are increased, e.g., through reduced helium heating or increased electron heating, the funnel amplifies the effect of radiation, since the increased radiative loss must be confined to the narrow funnel, resulting in a very high transition region pressure to support this radiative loss. Hence the funnel tends to create a positive feedback, causing a much larger increase in coronal densities than without the funnel. The large increase in coronal density in turn causes coronal $\alpha$-particles to become strongly collisionally coupled to electrons and protons. As a result, the $\alpha$-particles are too cold to escape into the solar wind. The helium abundance therefore builds up until helium is able to reduce the proton flux. The reduction in proton flux, while the total energy deposition is kept constant, means that an even larger fraction of the heat conducted downwards must be converted to radiation (since the enthalpy flux is reduced), causing a further jump in coronal densities. Hence the combined effect of the funnel and helium creates such a large increase in coronal densities that even if the heating parameters are subsequently reverted to their original values, the collisional coupling remains so strong that much of the energy is still conducted downwards, being lost as radiation, and a high-density corona with a high helium abundance, and a slow solar wind, persist.

In a funnel with a factor $f_{m 1}=20$ expansion enough energy is conducted into the transition region to support the observed fast solar wind mass flux. If the funnel is much narrower than this, will it still be possible to conduct down enough energy? The fast wind solution in Fig. 6 has a very small radiation loss, $\sim 2 \mathrm{~W}$, and the enthalpy flux uses almost all the energy conducted downwards. In Fig. 10 we compare the fast solution of Fig. 6 with the same model run with $f_{m 1}=200$. The solutions do not differ much; approximately the same amount of energy is converted downwards and the particle fluxes are only reduced by about $20 \%$. The coronal electron temperature is higher in the $f_{m 1}=200$ case, since a higher temperature is required to conduct a given heat flux down a narrower funnel. The solutions have similar densities, while asymptotic flow speeds are somewhat higher in the $f_{m 1}=200$ case, consistent with the somewhat lower particle fluxes. Hence even extremely narrow funnels seem to be able to provide the solar wind with the observed particle fluxes.

We have seen that in a funnel with a total expansion $f_{m 1} f_{m 2}=$ 100 , helium is not only readily pulled out of the chromosphere, but the frictional force is so strong that the helium flux is even locked to the hydrogen flux. Will heavier elements be pulled out of the chromosphere as well? Assuming that the species are mostly neutral in the chromosphere, whether particles are pulled 

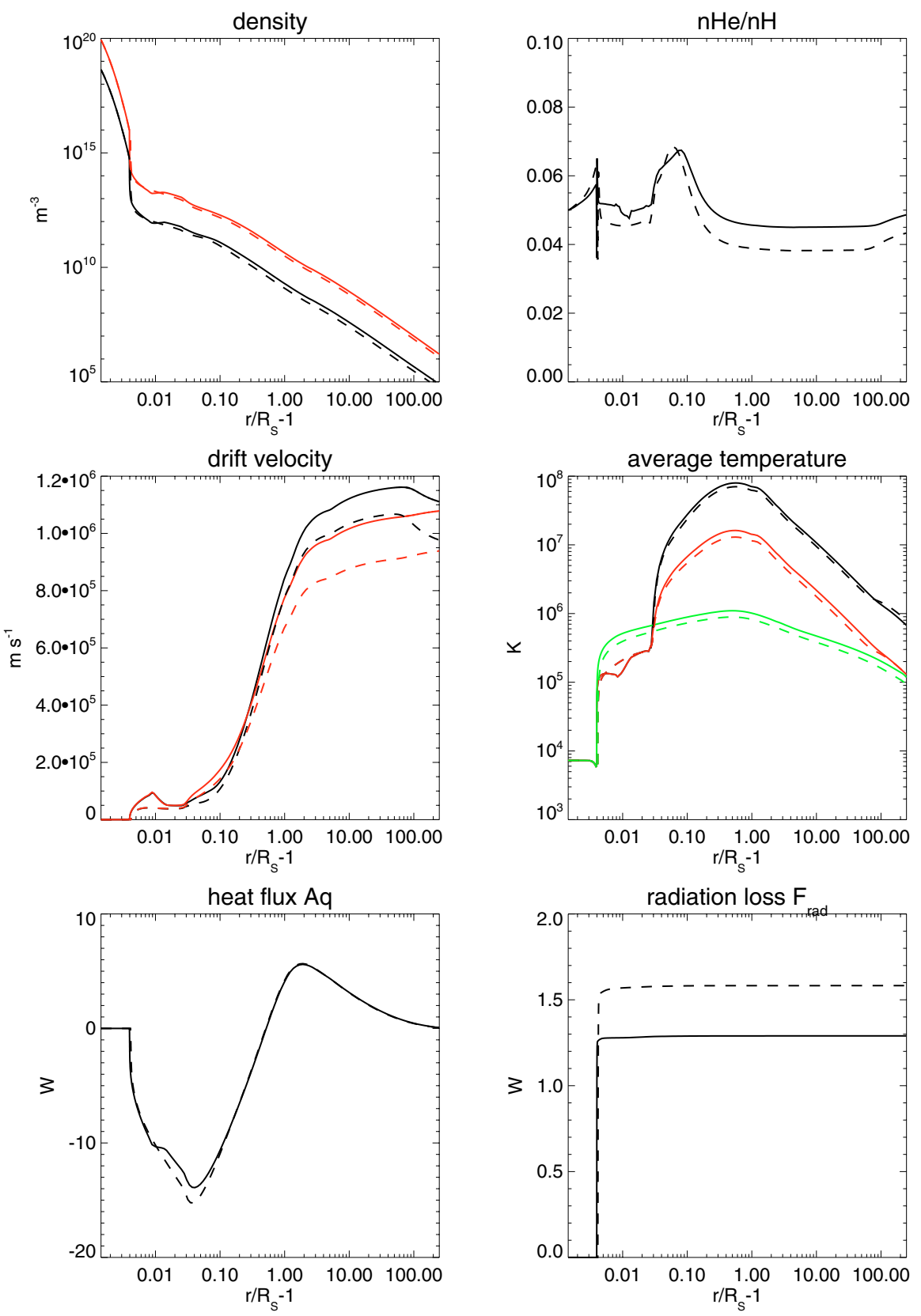

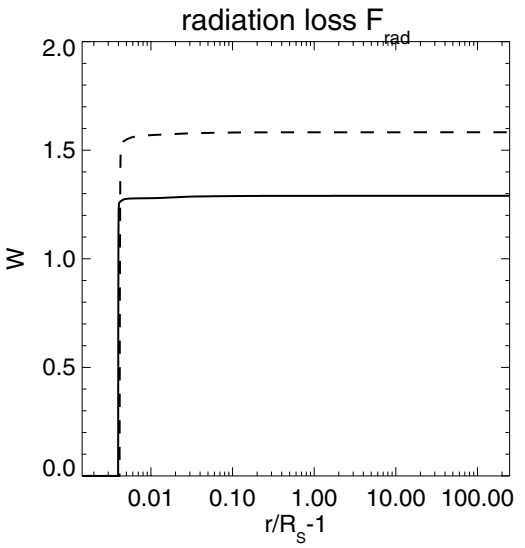

Fig. 10. Comparison of the funnel solution with $f_{m 1}=200$ (solid line) and the fast wind solution with $f_{m 1}=20$ in Fig. 6 (dashed). out of the chromosphere or not is determined by the parameter (Lie-Svendsen et al. 2003)

$\gamma \equiv \frac{m}{m_{\mathrm{p}}}\left(1-\frac{\hat{v} F_{\mathrm{H}}}{g}\right)-1$

where $m$ is the mass of the heavier element, $F_{\mathrm{H}}$ is the hydrogen flux density in the chromosphere, $g$ the gravitational acceleration, and

$\hat{v} \equiv \frac{2 \times 10^{-42} \mathrm{~kg} \mathrm{~m}^{3} \mathrm{~s}^{-1}}{m}$

is the collision frequency for neutral-neutral collisions divided by the hydrogen density. If $\gamma<0$ particles are readily pulled out, while for $\gamma>0$ gravitational settling will occur and the flux of minor particles will be very low. Scaling the observed hydrogen flux at $1 \mathrm{AU},\left(n_{\mathrm{H}} u_{\mathrm{H}}\right)_{\mathrm{E}}=2 \times 10^{12} \mathrm{~m}^{-2} \mathrm{~s}^{-1}$, to the chromosphere, the requirement $\gamma<0$ can be translated into

$f_{m 1} f_{m 2}>2.5\left(\frac{m}{m_{\mathrm{p}}}-1\right)$.

Thus for $m=4 m_{\mathrm{p}}$ (helium) we find $f_{m 1} f_{m 2}>7.5$; for oxygen we find $f_{m 1} f_{m 2}>38$; while for iron we get $f_{m 1} f_{m 2}>138$. In other words, of these only iron will be too heavy to be pulled up through the funnel with our chosen expansion factor.

\section{Summary}

The results show that in a low-density corona with a high-speed solar wind, the presence of a funnel at the base is of little importance for the solution, except that the funnel causes helium to be readily pulled out of the chromosphere. On the other hand, in solutions with a high-density corona, and corresponding slower solar wind, the funnel has a large impact on the solution: once the 
corona becomes collision-dominated, resulting in an increased downward heat flux and therefore a significantly increased radiative loss in the transition region, the presence of the funnel seems to amplify this process, causing an even higher coronal density and radiative loss. In fact, once a very high density corona has established, e.g., through reduced helium heating, the densities are so high that even if the heating parameters are reverted to those that will give a low-density corona and high-speed wind in the hole geometry, the funnel remains in the high-density state; the collisional coupling remains so large that protons and (particularly) $\alpha$-particles are unable to attain the high temperatures they need to escape. Hence we have, for the first time, been able to create two very different solar wind solutions with the same heating parameters and geometry, only differing in how the models were started.

The two co-existing solutions have properties at $1 \mathrm{AU}$ that resemble the observed properties of the high- and slow-speed solar wind, respectively. Hence it seems that if the solar wind originates in funnels, the high- and slow-speed wind are two modes that can be inherent to the system; high and low speed wind may originate from regions of the Sun that have very similar magnetic field configurations and are heated in similar ways. If the slow wind indeed is of this origin, an unavoidable consequence is that the coronal source of the slow wind must have a very high helium abundance.

In the parameter regime where the fast and slow modes can co-exist, a temporary change in the coronal heating process (e.g., a small flare) can trigger a transition between the two modes. However, the perturbation must be large; as shown above, switching from the fast to the slow solution we had to reduce the $\alpha$-particle heating to $1 / 4$ of its initial value, or add a very large electron heating term. In the former case a huge amount of helium accumulated in the corona in the intermediate state (see Fig. 5), while in the latter case the intermediate state did not have a high helium abundance, but instead a high coronal electron density and a much too large solar wind mass flux. The large perturbation required makes the co-existing modes stable, and the solar wind may not easily switch between them.

Although a huge increase in the helium abundance is not required to trigger the switch from the fast to the slow wind mode, helium is nevertheless essential in forming the slow wind mode, and the final coronal helium density must at least be of the same magnitude as the proton density in order to affect the force balance of protons (and hence the solar wind proton flux). It has proven difficult to infer the coronal helium abundance from observations. However, most studies indicate abundances of the order the photospheric abundance, $5 \%$ or even less in coronal holes (Laming \& Feldman 2001, 2003); and also in closed regions the abundance has been found to be less than approximately $10 \%$ (Gabriel et al. 1995; Laming \& Feldman 2001, 2003; Feldman et al. 2005). These values are too low to affect the proton flow and are therefore incompatible with the slow mode discussed here. As we have seen, we can produce solar wind models that do not violate these observational constraints. However, we have also demonstrated that it is easy for the Sun to produce much higher abundances. And if we are further constrained by the high coronal electron densities inferred from observations, $\alpha$-particles couple collisionally to protons and become too cold to easily escape the gravitational potential of the Sun, leading to accumulation of helium in the corona. In that case some fine tuning of model parameters is required in order to avoid very high helium abundances. This study therefore reconfirms previous studies, showing that it is easy to bring helium into the corona, but difficult to remove it from the corona. Based on classical transport theory we thus find it difficult to understand why the solar corona does not, at times, become helium rich.

Our findings are based on a model with specified coronal heating rates, for electrons, protons, and $\alpha$-particles: the energy flux from the Sun was specified as well as the damping length. In a more realistic model we may specify the energy flux from the Sun, but the energy transfer to the corona should depend on the plasma parameters; for instance, (a spectrum of) Alfvén waves entering the corona would certainly propagate and damp differently in a dense, slowly moving corona and in a low density, rapidly expanding corona. The transfer of energy, as heat and as work on the flow, will be quite different in the two cases. Would such a model, where a specified flux of Alfven waves is the energy source for the corona - solar wind system, also allow for co-existing solutions?

\section{References}

Axford, W. I., \& McKenzie, J. F. 1992, in Solar Wind Seven Colloq., ed. E. Marsch, \& R. Schwenn, 1

Axford, W. I., \& McKenzie, J. F. 1997, in Cosmic winds and the heliosphere, ed. J. R. Jokipii, C. P. Sonett, \& M. S. Giampapa (Tucson: University of Arizona), 31

Braginskii, S. I. 1965, in Rev. Plasma Phys., ed. M. A. Leontovich (New York: Consultants Bureau), 1, 205

Chodura, R., \& Pohl, F. 1971, Plasma Phys., 13, 645

Demars, H., \& Schunk, R. 1979, J. Phys. D. Appl. Phys., 12, 1051

Dorfi, E. A., \& Drury, L. O. 1987, J. Comput. Phys., 69, 175

Doschek, G. A., Warren, H. P., Laming, J. M., et al. 1997, ApJ, 482, L109

Dowdy, Jr., J. F., Rabin, D., \& Moore, R. L. 1986, Sol. Phys., 105, 35

Esser, R., Lie-Svendsen, Ø., Janse, A. M., \& Killie, M. A. 2005, ApJ, 629, L61

Feldman, U., Landi, E., \& Laming, J. M. 2005, ApJ, 619, 1142

Fisher, R. R., \& Guhathakurta, M. 1995, ApJ, 447, L139

Frazin, R. A., Cranmer, S. R., \& Kohl, J. L. 2003, ApJ, 597, 1145

Gabriel, A. H. 1976, Roy. Soc. Lond. Phil. Trans. Ser. A, 281, 339

Gabriel, A. H., Culhane, J. L., Patchett, B. E., et al. 1995, Adv. Space Res., 15, 63

Hackenberg, P., Marsch, E., \& Mann, G. 2000, A\&A, 360, 1139

Hansteen, V. H., \& Leer, E. 1995, J. Geophys. Res., 100, 21577

Hansteen, V. H., Leer, E., \& Holzer, T. E. 1997, ApJ, 482, 498

Janse, Å. M., Lie-Svendsen, Ø., \& Leer, E. 2005, J. Plasma Phys., 71, 611

Janse, A. M., Lie-Svendsen, Ø., \& Leer, E. 2006, Phys. Scr, T122, 66

Killie, M. A., Janse, Å. M., Lie-Svendsen, Ø., \& Leer, E. 2004, ApJ, 604, 842

Killie, M. A., Lie-Svendsen, Ø., \& Leer, E. 2005, ApJ, 632, L155

Kohl, J. L., Noci, G., Antonucci, E., et al. 1997, Sol. Phys., 175, 613

Kopp, R. A., \& Holzer, T. E. 1976, Sol. Phys., 49, 43

Koutchmy, S. 1977, Sol. Phys., 51, 399

Laming, J. M., \& Feldman, U. 2001, ApJ, 546, 552

Laming, J. M., \& Feldman, U. 2003, ApJ, 591, 1257

Leer, E., Holzer, T. E., \& Shoub, E. C. 1992, J. Geophys. Res., 97, 8183

Li, X. 2002, ApJ, 571, L67

Lie-Svendsen, Ø., Leer, E., \& Hansteen, V. H. 2001, J. Geophys. Res., 106, 8217

Lie-Svendsen, Ø., Leer, E., Hansteen, V. H., \& Holzer, T. E. 2002, ApJ, 566, 562

Lie-Svendsen, Ø., Hansteen, V. H., \& Leer, E. 2003, ApJ, 596, 621

Munro, R. H., \& Jackson, B. V. 1977, ApJ, 213, 874

Nakada, M. P. 1969, Sol. Phys., 7, 302

Rabin, D. 1991, ApJ, 383, 407

Schunk, R. W. 1977, Rev. Geophys. Space Phys., 15, 429

Spitzer, Jr., L., \& Härm, R. 1953, Phys. Rev., 89, 977

Tu, C.-Y., Zhou, C., Marsch, E., et al. 2005, Science, 308, 519

Wilhelm, K., Marsch, E., Dwivedi, B. N., et al. 1998, ApJ, 500, 1023

Withbroe, G. L. 1988, ApJ, 325, 442 